\title{
Quality and Pricing Decisions in a Two-Echelon Supply Chain with Nash Bargaining Fairness Concerns
}

\author{
Ji-cai Li $\mathbb{D}^{1}{ }^{1}$ Ji-hong Lu, ${ }^{1}$ Qi-liang Wang, ${ }^{2}$ and Changwen Li $\mathbb{D}^{3}$ \\ ${ }^{1}$ College of Xingzhi, Zhejiang Normal University, Jinhua 321004, China \\ ${ }^{2}$ School of Economics and Management, Zhejiang Normal University, Jinhua 321004, China \\ ${ }^{3}$ School of Economics, Huaibei Normal University, Huaibei 235000, China \\ Correspondence should be addressed to Ji-cai Li; leejicai@163.com
}

Received 30 January 2018; Accepted 28 March 2018; Published 9 May 2018

Academic Editor: Chuanxi Qian

Copyright (c) 2018 Ji-cai Li et al. This is an open access article distributed under the Creative Commons Attribution License, which permits unrestricted use, distribution, and reproduction in any medium, provided the original work is properly cited.

\begin{abstract}
Product quality and pricing, as the important competitive tools, play a key role in attracting consumers. In a supply chain, the decisions on product quality and pricing are usually interlinked and would influence the cooperation relation between the members, especially when they are fairness-concerned and have different bargaining power. However, linking the quality and pricing decisions to the decision-makers' behavioral factors such as fairness concern draws a little attention in the literature of supply chain management. This paper incorporates the members' fairness preference and bargaining power into the product quality and pricing decisions in a two-echelon supply chain, where the supplier offers core components with a certain quality level to the downstream manufacturer, who subsequently sells the final products in the end market. Both the supplier and the manufacturer are assumed to be fairness-concerned by adopting Nash bargaining solutions as their fairness reference points. We use gametheoretic models to analyze the equilibrium product quality and pricing strategies under the setting of integrated and decentralized supply chain, respectively. Detailed comparisons and sensitivity analysis are further conducted to examine the impacts of members' strengths of fairness concern, bargaining power, and decision structure on their equilibrium product quality and pricing strategies and corresponding payoffs.
\end{abstract}

\section{Introduction}

Product quality plays a pivotal role in ensuring customer satisfaction and enhancing competitive advantages (Shu and Wu [1]; Chai et al. [2]). From 1970s to 1990s, the unprecedented success of Japanese firms in industries such as automobiles and semiconductors mainly results from the superior quality and reliability of their products (Banker et al. [3]). Even in some contemporary high-tech markets, which are conventionally considered to exhibit strong network effects, quality prevails over network effects in affecting market share flows, and firms should still put more emphasis on the quality investment of their new products than on speed to market (Tellis et al. [4]; Li et al. [5]). Therefore, the issue of product quality and evaluation has received considerable attention from researchers and practitioners during the past three decades (Xu et al. [6]).

Meanwhile, consumers buy a product in consideration of not only its quality but also its selling price. Hence, firms' pricing strategy is an accompanying problem with quality issue. Furthermore, from a supply chain perspective, the nature of the coexistence of cooperation and competition between channel members also usually results in the qualityrelated investment and pricing decisions being interlinked. They work together to boost market demand by various demand-enhancing efforts, while the efforts (e.g., investment in technology/innovation, which can improve product quality) implemented and afforded by either firm can benefit all members. As such, the upstream firm investing in qualityimproving technology/innovation may prefer to charge a higher wholesale price to the downstream firm; reversely the latter being closer to the end market might prefer a higher selling price (Gurnani et al. [7]).

In such a case, unreasonably setting wholesale and resale price, respectively, by the supplier of core components and the manufacturer of final products, may either lead the supplier to underinvest in quality improvement or even cause channel 
members to terminate cooperation. This is much more so when the members in a supply chain have fairness preference and different bargaining power. For instance, in January 2017, Apple launched a hefty \$1 billion lawsuit against Qualcomm which is the key chips supplier for iPhones, arguing that Qualcomm deliberately overcharged for its technologies investment. The lawyers for Apple even said: "It amounts to a scheme of extortion that allows Qualcomm unfairly to maintain and entrench its existing monopoly." After that, Apple has also partnered with Intel in an effort to reduce cost and lessen its dependence on the chipmaker (see Looper [8] for details).

To the best of our knowledge, so far there are few studies which link the quality and pricing decisions with the decision-makers' fairness preference in the context of a multitier supply chain. While in the area of behavioral economics, a lot of studies mentioned that there is a high incidence of cases in which firms are motivated by concerns of fairness and sacrifice their own margins to punish their counterpart when unfairness is perceived (Kahneman et al. [9]; Fehr and Schmidt [10]; Kumar [11]; Scheer et al. [12]). This implies that channel members usually have fairness preference; that is, they seek to maximize a utility function that accounts for their own monetary payoff as well as the fair allocation of the channel's overall profit. Moreover, the conclusion that the decision-making of supply chain members will be profoundly influenced by their fairness preference has been validated by many experimental studies such as Loch and Wu [13] and Katok and Pavlov [14]. Hence, it is very necessary to incorporate the members' fairness preference into the supply chain with quality and pricing decisions.

In this paper, we consider a two-echelon supply chain consisting of a supplier and a manufacturer, where the supplier invests in technology and offers core components with a certain quality level and wholesale price to the manufacturer, who then processes core components into final products and sets the resale price in the end market. We assume that both the supplier and the manufacturer are fairness-concerned by adopting Nash bargaining solutions as their fairness reference points. Both an integrated and a decentralized supply chain are studied to further examine how fairness concern, bargaining power, and decision structures influence two members' quality and pricing strategies and their performances.

We divide the market into two types, that is, the pricesensitive and quality-sensitive market, according to whether selling price is sufficiently more or less important than quality to consumers (the formal definition is given below Theorem 1). Our research shows the following main results. First, the product quality, market demand, and overall profit of the supply chain under decentralized setting are correspondingly not higher than those under integrated setting, no matter what the market type is and whether the members are fairness-concerned or not. And in a quality-sensitive market, all their differences between the integrated and decentralized settings are always increasing in the market desire to innovate to improve product quality. Second, for a decentralized supply chain, the supplier's fairness preference will make the product quality, demand, and overall profit much lower in both types of market, while the manufacturer's fairness preference impacts them only in a quality-sensitive market. Third, the equilibrium wholesale price and selling price decrease with the manufacturer's strength of fairness concern, except that the selling price in a price-sensitive market is irrelevant to it, whereas the supplier's more strength of fairness concern and bargaining power are more likely to lead to a higher equilibrium wholesale price and selling price, unless when the manufacturer's strength of fairness concern and quality-related fixed cost coefficient are small enough. At last, another interesting finding is that, in a quality-sensitive market, one member's sufficiently smaller strength of fairness concern than his/her counterpart's is more conducive to lead both him/her and his/her counterpart to get higher profits as his/her bargaining power increases, which in turn results in higher overall profit of the supply chain.

The rest of the paper is organized as follows. Section 2 reviews the related literature. Section 3 presents the problem description and some preliminaries. Section 4 formulates the models and studies the equilibrium quality and pricing policies for the integrated and decentralized scenarios. Section 5 compares these equilibrium values and discuss the impacts of the members' fairness concern, bargaining power, and decision structure on the equilibrium strategies and associated payoffs. Section 6 concludes the paper.

\section{Literature Review}

Our research is mainly related to two streams of literature: product quality and pricing strategies and supply chain decisions with fairness concern. To highlight our contributions, we review the representative literature in these two streams.

The first stream is related to the literature on optimal quality and pricing strategies. One aspect of these studies focus on quality investment and pricing decisions for substitutable products, respectively, from multiple horizontal firms. Banker et al. [3] developed three different models of oligopolistic competition on product quality and selling price aiming to investigate the implication of competitive intensity on equilibrium levels of quality. Matsubayashi [15] explored price and quality decisions of two competitive identical firms and focused on utilizing two possible business strategies, product differentiation, and vertical integration with another complementary firm, to overcome the competition. Matsubayashi and Yamada [16] analyzed how the asymmetry in consumer loyalty between two firms affects their price and quality competition.

Recently, some researchers studied product quality and pricing decisions in the context of a multitier supply chain. $\mathrm{Yu}$ and $\mathrm{Ma}$ [17] studied the impact of decision sequence of pricing and quality in a decentralized assembly system, consisting of two suppliers who decide on wholesale price and quality for their components, and one manufacture sets selling price of the final product. Seifbarghy et al. [18] considered pricing and quality decisions in a two-level supply chain consisting of a manufacturer and a retailer under four different conditions. Wang et al. [19] explored the interaction of channel structure with price- and quality-based competition between two manufacturers who are asymmetric in consumer loyalty. Xie et al. [20] considered the selection of channel structure and quality improvement strategies of 
two competing supply chains, which offer a product at the same selling price but compete on quality. Xie et al. [21] studied quality investment and selling price decisions of a make-to-order supply chain where both the supplier and the manufacturer are risk averse. Giri et al. [22] analyzed the ordering, pricing, and quality management strategies for a single product in a three-echelon supply chain, where the market demand is price and product quality dependent, and investigated the performances of coordinating the supply chain system through two strategies, that is, sub-supply chain coordination strategy and two-level retail fixed mark-up (RFM) strategy. Chen et al. [23] investigated price and quality decisions in dual-channel supply chains, in which a single product is delivered through a retail channel, a direct channel, or a dual channel with both retail and direct channels, and they demonstrated that quality improvement can be realized when a new channel is introduced.

The second related stream is the studies on supply chain decisions with fairness concern. It is pioneered by Cui et al. [24] who incorporated the concept of fairness in a dyadic channel to investigate how fairness affects channel coordination. Since then, many researchers have introduced members' fairness concern into different supply chain models. The existing studies belong to one of two types. One is about peer-induced fairness, which characterizes the fairness preferences of multiple agents who are in similar circumstances. Representative literature of this type includes Chen and Cui [25], $\mathrm{Li}$ and Jain [26], Du et al. [27], and Nie and Du [28]. The other is about distributional fairness, which is more closely related to this paper and characterizes the fairness preferences of agents who are usually at vertically different echelons in a supply chain and concerned about the fair distribution of total benefits. Two utility functions, that is, F-S model developed by Fehr and Schmidt [10] and L-W model developed by Loch and Wu [13], are most commonly employed to describe the distributional fairness preference. Besides Cui et al. [24], the studies of Katok et al. [29] and Q.-H. Li and B. Li [30] also adopt F-S model. Katok et al. [29] studied the performance of wholesale pricing when the channel members' fairness concerns are private information. Q.-H. Li and B. Li [30] considered a dual-channel supply chain where a manufacturer produces a single product and sells the product through a direct channel, as well as a traditional retail channel in which the retailer has fairness concerns and provides value-added services to consumers. In virtue of L-W model, Ho et al. [31] examined how distributional and peer-induced fairness might interact in the design of wholesale price contract and influence the supply chain members' economic outcomes. However, these two fairness utility models have a common limitation. That is, they cannot capture players' power and contribution endogenously, which affects the fairness perception. To address this issue, Du et al. [32] innovatively used Nash bargaining solutions as channel members' fairness reference points and examined the impacts of fairness concerns on the optimal decisions in newsvendor problem for a dyadic supply chain. Chen et al. [33] studied the pricing and ordering issues in a dyadic supply chain consisting of a supplier and a budget-constrained retailer with fairness concern. They adopted the same approach as
Du et al. [32] to explore how the retailer's fairness concern and buyback guarantee financing mode affect two members' equilibrium strategies.

To the best of our knowledge, so far only Guo and Jiang [34] linked the quality and pricing issues to the widespread fairness concern of human agents. They examined a firm's product quality and pricing decisions when facing consumers who have distributional fairness concern. Our paper differs from theirs in at least three aspects. First, we study quality and pricing decisions in a supplier-manufacturer supply chain where the supplier decides on the product quality and wholesale price and the retailer as a follower sets the selling price to consumers. However, they only focus on a single firm's product quality and selling price decisions, not on the context of a supply chain. Second, they consider the consumers' fairness preference but the firm as the unique decision maker is fairness-neutral, while in our paper both the supplier and the retailer have fairness preference and focus on how the fairness concerns of them influence their own equilibrium product quality and pricing decisions and performances. Third, we use Nash bargaining solution as the fairness reference point to measure the members' fairness preference and capture their power and contribution endogenously, but they do not consider the decision-marker's power and contribution.

\section{Preliminaries}

3.1. Problem Description. We consider a two-echelon supply chain consisting of a supplier and a manufacturer. The supplier produces a core component and provides it to the manufacturer through a wholesale price contract. After receiving the core components, the manufacturer further processes them into final products to be sold to consumers. One unit core component can be processed into one unit final product. Like most studies on product quality decision in a supply chain (e.g., Xie et al. [21]; Yu and Ma [17]), we assume that the manufacturer cannot change the quality of core components, and the quality of final products is determined by the core components.

Following the definition of quality in Banker et al. [3] and Jerath et al. [35], we use the term of "quality" to refer to both design and conformance quality characteristics which are of interest to consumers. Consumers can readily discern the quality level of products prior to purchase. In economics, this kind of product is named as search good (e.g., consumer electronics; see Nelson [36] for details). Therefore, not only selling price but also quality level will be taken into account when consumers decide whether to buy a product.

Both the supplier and the manufacturer are assumed to be fairness-concerned. That is, they not only are concerned about their self-profit, but also care about the distributional fairness of the supply chain profit. Nash bargaining solution is used as the mutually agreed fairness reference point, since it has several desirable properties and satisfies a set of axioms which are attractive to defining fairness. This approach has also been successfully applied in Du et al. [32], Chen et al. [33], and so forth.

We will study both a vertically integrated and a decentralized supply chain. The event sequence can be described 
as follows. In the integrated scenario, the supplier and the manufacturer cooperate together to select the optimal quality level and selling price of the final products, whereas in the decentralized scenario, the supplier moves first to set quality level and wholesale price of the core components, and the manufacturer moves later to decide the selling price of the final products. After that, the demand from the end market is realized based on the quality level and selling price, and then the two members accordingly organize their production to satisfy the demand.

3.2. Notation and Assumption. The symbols used in the paper are presented in Notations.

As depicted in Section 3.1, p, q, and $w$ are decision variables; they all should be nonnegative; other parameters are exogenous and known to both members. It is assumed that $p>w+v_{M}$ and $w>v_{S}+\varepsilon q$. These inequalities ensure that each member can make a positive margin.

The demand for the final products is assumed to be linear in selling price $p$ and quality $q$. That is,

$$
D=a+\alpha q-\beta p
$$

where $a, \alpha$, and $\beta$ are positive constants and $a>\beta\left(v_{S}+\right.$ $v_{M}$ ) such that demand is always positive. Similar demand functions have been widely used in related studies, for example, Banker et al. [3], Xie et al. [21], and Giri et al. [22].

In addition, we assume that the quality level selected by the supplier affects his total cost in two ways. (Throughout this paper, we view the supplier as a "male" and the manufacturer as a "female".) First, the variable production cost per unit is affected linearly by the quality level; that is, $v_{S}+\varepsilon q$, where $v_{S}>0$ and $\varepsilon \geq 0$. Second, investment in a quality improvement program will affect his fixed cost $c q^{2}$ with $c>0$, which is increasing and convex in $q$. Since the manufacturer's production does not affect the quality level of components and final products, her total cost is irrelevant to product quality, and the variable production cost per unit for her is $v_{M}>0$. Such cost structures have been used in Matsubayashi and Yamada [16], Yu and Ma [17], and Wang et al. [19].

\subsection{Modelling Fairness-Concerned Firms with Nash Bargain-} ing Reference. For a fairness-concerned decision maker, its utility depends on both its realized monetary payoff and the gap to its fairness reference point. A linear utility function is often used as follows:

$$
u_{i}^{F}=\pi_{i}+\lambda_{i}\left(\pi_{i}-\bar{\pi}_{i}\right), \quad i=S, M,
$$

where the subscript $i=S, M$ represents the supplier and the manufacturer, $\pi_{i}$ and $\bar{\pi}_{i}$ denote the $i$ 's realized profit and fairness reference point, respectively. $\lambda_{i} \geq 0$ captures $i$ 's strength of fairness concern, and the higher $\lambda_{i}$ is, the more fairness-concerned it is. $\lambda_{i}=0$ means the firm $i$ is fairnessneutral.

As stated in Section 2, F-S model and L-W model are commonly used as fairness-concerned utility models. For these two utility models, each member in the supply chain views the other's realized profit as its own fairness reference. The difference is that L-W model considers relationship as well as status (Loch and Wu [13]) but F-S model does not (Fehr and Schmidt [10]). However, both these two models do not capture players' power and contribution endogenously ( Du et al. [32]). With this in mind, we employ Nash bargaining solution as both members' mutually agreed fairness reference point, which was also used by Du et al. [32] and Chen et al. [33].

According to Nash's axiomatic definition (Nash [37]; Harsanyi and Selten [38]), Nash bargaining solution $\left(\bar{\pi}_{S}, \bar{\pi}_{M}\right)$ is the monetary payoff pair $\left(\pi_{S}, \pi_{M}\right)$ that maximizes the Nash product in the following form:

$$
\begin{array}{ll}
\max _{\pi_{S}, \pi_{M}} & \left(u_{S}^{F}\right)^{\tau}\left(u_{M}^{F}\right)^{1-\tau} \\
\text { s.t. } & \pi_{S}+\pi_{M}=\pi_{S C} \\
& \pi_{S} \geq 0, \pi_{M} \geq 0,
\end{array}
$$

where $\pi_{\mathrm{SC}}$ is the total profit of the supply chain, $0<\tau<1$ denotes the supplier's bargaining power, and $(1-\tau)$ denotes the manufacturer's.

Following a similar way in Du et al. [32], the supplier's and manufacturer's fairness reference points, respectively, are

$$
\begin{gathered}
\bar{\pi}_{S}=\frac{\tau\left(1+\lambda_{S}\right)}{1+\tau \lambda_{S}+(1-\tau) \lambda_{M}} \pi_{\mathrm{SC}}, \\
\bar{\pi}_{M}=\frac{(1-\tau)\left(1+\lambda_{M}\right)}{1+\tau \lambda_{S}+(1-\tau) \lambda_{M}} \pi_{S C} .
\end{gathered}
$$

Hence, the utility functions of the two fairness-concerned firms can be expressed as follows:

$$
\begin{aligned}
& u_{S}^{F}=\left(1+\lambda_{S}\right) \pi_{S}-\frac{\tau \lambda_{S}\left(1+\lambda_{S}\right)}{1+\tau \lambda_{S}+(1-\tau) \lambda_{M}} \pi_{\mathrm{SC}}, \\
& u_{M}^{F}=\left(1+\lambda_{M}\right) \pi_{M}-\frac{(1-\tau) \lambda_{M}\left(1+\lambda_{M}\right)}{1+\tau \lambda_{S}+(1-\tau) \lambda_{M}} \pi_{\mathrm{SC}} .
\end{aligned}
$$

\section{The Optimal Decisions}

4.1. The Optimal Decisions in an Integrated Supply Chain. Now we consider a vertically integrated setting where both the supplier and the manufacturer are owned by the same firm. The solution of this setting can be used as a benchmark to facilitate our analysis. Additionally, there are many practical supply chains which accord with this setting. For example, the Gree group, a leading manufacturer in global air-conditioner industry, procures compressor as a key component of air-conditioner from its subfirm, Zhuhai Lingda Compressor Company.

Since the two members cooperate as a whole, they are willing to jointly maximize the total profit of the supply chain, regardless of whether they are fairness-concerned or not. Clearly, there are only two decision variables, namely, $p$ and $q$, and the objective function for such integrated setting is

$$
\begin{aligned}
\max & \pi_{\mathrm{SC}}^{C}(p, q) \\
& =\left(p-v_{M}-v_{S}-\varepsilon q\right)(a+\alpha q-\beta p)-c q^{2} .
\end{aligned}
$$


Taking the second derivatives of $\pi_{\mathrm{SC}}^{\mathrm{C}}(p, q)$, the Hessian matrix of $\pi_{\mathrm{SC}}^{\mathrm{C}}(p, q)$ is given as

$$
\begin{aligned}
H^{C} & =\left[\begin{array}{ll}
\frac{\partial^{2} \pi_{S C}^{C}(p, q)}{\partial p^{2}} & \frac{\partial^{2} \pi_{S C}^{C}(p, q)}{\partial p \partial q} \\
\frac{\partial^{2} \pi_{S C}^{C}(p, q)}{\partial q \partial p} & \frac{\partial^{2} \pi_{S C}^{C}(p, q)}{\partial q^{2}}
\end{array}\right] \\
& =\left[\begin{array}{cc}
-2 \beta & \alpha+\beta \varepsilon \\
\alpha+\beta \varepsilon & -2(c+\alpha \varepsilon)
\end{array}\right] .
\end{aligned}
$$

To ensure the existence and uniqueness of equilibrium, it is assumed throughout the paper that

$$
4 \beta c-(\alpha-\beta \varepsilon)^{2}=4 \beta c-T^{2}>0 .
$$

Theorem 1. The equilibrium solution for quality level and selling price $\left(q^{C *}, q^{C *}\right)$ in an integrated supply chain is specifically given as follows:

(i) When $T<0$, then $q^{C *}=0, p^{C *}=\left(a+\beta\left(v_{M}+v_{S}\right)\right) / 2 \beta$;

(ii) When $T \geqslant 0$, then $q^{C *}=T\left[a-\beta\left(v_{M}+v_{S}\right)\right] /\left(4 \beta c-T^{2}\right)$, $p^{C *}=v_{M}+v_{S}+(2 c+\varepsilon T)\left[a-\beta\left(v_{M}+v_{S}\right)\right] /\left(4 \beta c-T^{2}\right)$.

Proof. See Appendix.

Theorem 1 tells us that the above equilibrium is characterized by the sign of $T$, that is, the demand sensitivity to quality relative to selling price (normalized by the variable production cost of quality). Thus, the market can be divided into two types: the price-sensitive market when $T<0$ (i.e., $\beta>\alpha / \varepsilon$, or selling price is more important than quality to consumers) and the quality-sensitive market when $T \geq 0$ (i.e., $\alpha / \varepsilon \geq \beta$, or quality is more important than selling price to consumers). Only in a quality-sensitive market will the integrated supply chain have incentives to improve the quality level. However, in a price-sensitive market, the equilibrium quality level is necessarily zero and independent of the value of $T$. As we will see next, this observation is still valid for a decentralized supply chain no matter whether its members are fairness-concerned or not.

Substituting the solution into (1) and (7), we obtain the corresponding demand and profit yielded by the integrated supply chain at the equilibrium, which are listed in Tables 1 and 2 .

\subsection{The Optimal Decisions in a Decentralized Supply Chain.} Recent decades have witnessed increasing economic globalization. In such backdrop, components outsourcing is more popular and nearly becomes a normal production practice for manufacturers in many industries. Hence, most suppliers of components and the manufacturers of final products do not belong to the same company; they often make decisions independently to maximize their own objectives.

This section will consider a decentralized setting in which the supplier and the manufacturer make their decisions separately. The supplier, as the leader, plays a Stackelberg game with the manufacturer. That is, the supplier first sets his quality $q$ and wholesale price $w$ of the core components by taking the manufacturer's best response into account; then the manufacturer, after observing the supplier's decision, decides the selling price $p$ of the final products for consumers. This event sequence is a common assumption for the pricing and quality decisions in decentralized supply chains (Wang et al. [19]).

In such a decentralized supply chain, the profit functions of the manufacturer and the supplier, respectively, are

$$
\begin{aligned}
\pi_{M}(p) & =\left(p-w-v_{M}\right)(a+\alpha q-\beta p), \\
\pi_{S}(q, w) & =\left(w-v_{S}-\varepsilon q\right)(a+\alpha q-\beta p)-c q^{2} .
\end{aligned}
$$

Since the supplier and manufacturer are assumed to be fairness-concerned, both of them aim to maximize their fairness-related utilities, respectively, given in (5) and (6). Specifically for the manufacturer, substituting (7) and (10) into (6) yields her utility function as follows:

$$
\begin{aligned}
& u_{M}^{F}(p)=\left(1+\lambda_{M}\right)\left(p-w-v_{M}\right)(a+\alpha q-\beta p) \\
& -\frac{(1-\tau) \lambda_{M}\left(1+\lambda_{M}\right)}{1+\tau \lambda_{S}+(1-\tau) \lambda_{M}}\left[\left(p-v_{M}-v_{S}-\varepsilon q\right)\right. \\
& \left.\cdot(a+\alpha q-\beta p)-c q^{2}\right] .
\end{aligned}
$$

Taking the first- and second-order derivatives of $u_{M}^{F}(p)$ with respect to $p$, we get

$$
\begin{aligned}
& \frac{d u_{M}^{F}(p)}{d p}=\left(1+\lambda_{M}\right)\left(\beta\left(w-v_{S}-\varepsilon q\right)\right. \\
& \left.\quad+\frac{\left(1+\tau \lambda_{S}\right)\left[a+\alpha q+\beta\left(v_{M}+v_{S}+\varepsilon q-2 p\right)\right]}{1+\tau \lambda_{S}+(1-\tau) \lambda_{M}}\right), \\
& \frac{d^{2} u_{M}^{F}(p)}{d p^{2}}=\frac{-2 \beta\left(1+\lambda_{M}\right)\left(1+\tau \lambda_{S}\right)}{1+\tau \lambda_{S}+(1-\tau) \lambda_{M}}<0 .
\end{aligned}
$$

Hence, the manufacturer's utility function is strictly concave in $p$, and solving the first-order condition gives her best response function:

$$
\begin{aligned}
p^{F *}(q, w)= & \frac{a+\alpha q+\beta\left(w+v_{M}\right)}{2 \beta} \\
& +\frac{(1-\tau) \lambda_{M}\left(w-v_{S}-\varepsilon q\right)}{2\left(1+\tau \lambda_{S}\right)} .
\end{aligned}
$$

Specially, if the manufacturer is fairness-neutral (i.e., $\lambda_{M}=0$ ), her best response function is

$$
p^{N *}(q, w)=\frac{a+\alpha q+\beta\left(w+v_{M}\right)}{2 \beta} .
$$

Property 2. For any given quality level $q$ and wholesale price $w$,

(i) $\partial p^{F *}(q, w) / \partial \lambda_{M}>0, \partial p^{F *}(q, w) / \partial \lambda_{S}<0, \partial p^{F *}(q, w) /$ $\partial \tau<0$.

(ii) $\partial p^{F *}(q, w) / \partial v_{S}<0, \partial p^{F *}(q, w) / \partial \varepsilon<0$.

(iii) $p^{F *}(q, w) \geq p^{N *}(q, w)$. 
TABLE 1: The equilibrium solutions under different scenarios when $T<0$.

\begin{tabular}{|c|c|c|c|}
\hline & Integrated supply chain & $\begin{array}{l}\text { Decentralized supply chain with } \\
\text { fairness-neutral members }\end{array}$ & $\begin{array}{l}\text { Decentralized supply chain with } \\
\text { fairness-concerned members }\end{array}$ \\
\hline Selling price & $p^{C *}=\frac{a+\beta\left(v_{M}+v_{S}\right)}{2 \beta}$ & $p^{N *}=\frac{3 a+\beta\left(v_{M}+v_{S}\right)}{4 \beta}$ & $p^{F *}=\frac{\psi a+\beta(2 \xi-\psi)\left(v_{M}+v_{S}\right)}{2 \beta \xi}$ \\
\hline Wholesa & -- & $w^{N *}=\frac{a-\beta\left(v_{M}-v_{S}\right)}{2 \beta}$ & $w^{F *}=\frac{\kappa\left(a-\beta v_{M}\right)+\beta(\xi-\kappa) v_{S}}{\beta \xi}$ \\
\hline Quality level & $q^{C *}=0$ & $q^{N *}=0$ & $q^{F *}=0$ \\
\hline Demand & $D^{C *}=\frac{1}{2}\left[a-\beta\left(v_{M}+v_{S}\right)\right]$ & $D^{N *}=\frac{1}{4}\left[a-\beta\left(v_{M}+v_{S}\right)\right]$ & $D^{F *}=\frac{2 \xi-\psi}{2 \xi}\left[a-\beta\left(v_{M}+v_{S}\right)\right]$ \\
\hline The supplier's profit & -- & $\pi_{S}^{N *}=\frac{\left[a-\beta\left(v_{M}+v_{S}\right)\right]^{2}}{8 \beta}$ & $\pi_{S}^{F *}=\frac{(2 \xi-\psi) \kappa}{2 \beta \xi^{2}}\left[a-\beta\left(v_{S}+v_{M}\right)\right]^{2}$ \\
\hline $\begin{array}{l}\text { The manufacture's } \\
\text { profit }\end{array}$ & -- & $\pi_{M}^{N *}=\frac{\left[a-\beta\left(v_{M}+v_{S}\right)\right]^{2}}{16 \beta}$ & $\pi_{M}^{F *}=\frac{(2 \xi-\psi)(\psi-2 \kappa)}{4 \beta \xi^{2}}\left[a-\beta\left(v_{S}+v_{M}\right)\right]^{2}$ \\
\hline $\begin{array}{l}\text { The whole channel's } \\
\text { profit }\end{array}$ & $\pi_{\mathrm{SC}}^{\mathrm{C} *}=\frac{\left[a-\beta\left(v_{M}+v_{S}\right)\right]^{2}}{4 \beta}$ & $\pi_{\mathrm{SC}}^{N *}=\frac{3}{16 \beta}\left[a-\beta\left(v_{M}+v_{S}\right)\right]^{2}$ & $\pi_{\mathrm{SC}}^{F *}=\frac{(2 \xi-\psi) \psi}{4 \beta \xi^{2}}\left[a-\beta\left(v_{S}+v_{M}\right)\right]^{2}$ \\
\hline
\end{tabular}

TABLE 2: The equilibrium solutions under different scenarios when $T \geq 0$.

\begin{tabular}{|c|c|c|c|}
\hline & Integrated supply chain & $\begin{array}{l}\text { Decentralized supply chain with } \\
\text { fairness-neutral members }\end{array}$ & ss-concerned m \\
\hline Sellin & $\begin{array}{c}p^{C *}=v_{M}+v_{S}+ \\
\frac{(2 c+\varepsilon T)\left[a-\beta\left(v_{M}+v_{S}\right)\right]}{4 \beta c-T^{2}}\end{array}$ & $p^{N *}=v_{M}+v_{S}+\frac{(6 c+\varepsilon T)\left[a-\beta\left(v_{M}+v_{S}\right)\right]}{8 \beta c-T^{2}}$ & $p^{F *}=v_{M}+v_{S}+\frac{(2 c \psi+\varepsilon T)\left[a-\beta\left(v_{M}+v_{S}\right)\right]}{4 \beta c \xi-T^{2}}$ \\
\hline Wholesale price & 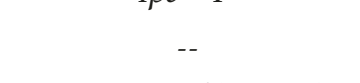 & $w^{N *}=v_{S}+\frac{(4 c+\varepsilon T)[a}{8 \beta c}$ & $w^{F *}=v_{S}+\frac{(4 c \kappa+\varepsilon T)\left[a-\beta\left(v_{M}+v_{S}\right)\right]}{4 \beta c \xi-T^{2}}$ \\
\hline Quali & $q^{C *}=\frac{T\left[a-\beta\left(v_{M}+v_{S}\right)\right]}{4 \beta c-T^{2}}$ & $q^{N *}=\frac{T\left[a-\beta\left(v_{M}+v_{S}\right)\right]}{8 \beta c-T^{2}}$ & $q^{F *}=\frac{T\left[a-\beta\left(v_{M}+v_{S}\right)\right]}{4 \beta c \xi-T^{2}}$ \\
\hline Demand & $D^{C *}=\frac{2 \beta c\left[a-\beta\left(v_{M}+v_{S}\right)\right]}{4 \beta c-T^{2}}$ & $D^{N *}=\frac{2 \beta c\left[a-\beta\left(v_{M}+v_{S}\right)\right]}{8 \beta c-T^{2}}$ & $D^{F *}=\frac{2 \beta c(2 \xi-\psi)\left[a-\beta\left(v_{M}+v_{S}\right)\right]}{4 \beta c \xi-T^{2}}$ \\
\hline r's profit & -- & $\pi_{S}^{N *}=\frac{c\left[a-\beta\left(v_{M}+v_{S}\right)\right]^{2}}{8 \beta c-T^{2}}$ & $\begin{array}{c}\pi_{S}^{F *}= \\
c\left[8 \beta c(2 \xi-\psi) \kappa-T^{2}\right]\left[a-\beta\left(v_{M}+v_{S}\right)\right]^{2} \\
\end{array}$ \\
\hline $\begin{array}{l}\text { The ma } \\
\text { profit }\end{array}$ & -- & $\pi_{M}^{N *}=\frac{4 \beta c^{2}\left[a-\beta\left(v_{M}+v_{S}\right)\right]^{2}}{\left(8 \beta c-T^{2}\right)^{2}}$ & $\begin{array}{c}\frac{\left(4 \beta c \xi-T^{2}\right)^{2}}{\pi_{M}^{F *}=} \\
\frac{4 \beta c^{2}(2 \xi-\psi)(\psi-2 \kappa)\left[a-\beta\left(v_{M}+v_{S}\right)\right]^{2}}{\left(4 \beta c \xi-T^{2}\right)^{2}}\end{array}$ \\
\hline $\begin{array}{l}\text { The whole channel's } \\
\text { profit }\end{array}$ & $\pi_{\mathrm{SC}}^{C *}=\frac{c\left[a-\beta\left(v_{M}+v_{S}\right)\right]^{2}}{4 \beta c-T^{2}}$ & $\pi_{\mathrm{SC}}^{N *}=\frac{c\left(12 \beta c-T^{2}\right)\left[a-\beta\left(v_{M}+v_{S}\right)\right]^{2}}{\left(8 \beta c-T^{2}\right)^{2}}$ & $\begin{array}{c}\pi_{\mathrm{SC}}^{F *}= \\
\frac{c\left[4 \beta c(2 \xi-\psi) \psi-T^{2}\right]\left[a-\beta\left(v_{M}+v_{S}\right)\right]^{2}}{\left(4 \beta c \xi-T^{2}\right)^{2}}\end{array}$ \\
\hline
\end{tabular}

Proof. It is straightforward and is therefore omitted.

Property 2 indicates that when the quality $q$ and wholesale price $w$ of core components are given, the manufacturer's optimal selling price increases with her fairness concern coefficient $\lambda_{M}$, whereas it decreases with the supplier's fairness concern coefficient $\lambda_{S}$. That is, the more fairness-concerned the manufacturer (supplier) is, the higher (lower) selling price will be set. And the selling price decreases with the supplier's bargaining power $\tau$ (or, say, increases with the manufacturer's bargaining power $1-\tau$ ). These insights make sense, because when the manufacturer's bargaining power or her strength of fairness concern increases (similarly, the supplier's strength of fairness concern decreases), for any given quality and wholesale price of core components, she will be more aggressive so as to set a higher selling price for final products to obtain a larger margin.

Additionally, Property 2 shows that for any given quality and wholesale price of core components provided by the supplier, the manufacturer's optimal selling price is decreasing in the supplier's variable production cost parameters (i.e., $v_{S}$ and $\varepsilon$ ). This is different from the results in a fairness-neutral channel, where her optimal selling price is independent of the supplier's variable production cost (see (15)). This is because the manufacturer in a fairness-neutral channel only cares about her own profit; however, in a fairness-concerned channel she also pays attention to the fair distribution of the channel's overall profit. It is clear that for any given quality and wholesale price, the higher variable production cost the supplier has, the lower margin he can get. Therefore, the manufacturer will set a lower selling price and also get a lower 
margin to ensure a relatively fair distribution of the channel's overall profit.

Besides, Property 2 tells us that, given any $q$ and $w$ selected by the supplier, the manufacturer's optimal selling price in a fairness-concerned channel is always greater than that in its fairness-neutral counterpart. This is because the positive impacts of parameters denoting her own characteristics (i.e., $\lambda_{M}$ and $1-\tau$ ) on her pricing decision dominate the negative impacts of the supplier's parameters (i.e., $\lambda_{S}, v_{S}$ and $\varepsilon$ ).

Having characterized the manufacturer's best response, next we analyze the supplier's decisions. Substituting the supplier's profit in (11), the channel's overall profit in (7), and the manufacturer's best response function in (14) into (5), the supplier's utility is

$$
\begin{aligned}
& u_{S}^{F}(q, w)=\frac{1+\lambda_{S}}{4}\left[\frac{\left(2+\tau \lambda_{S}\right)\left(w-v_{S}-\varepsilon q\right)}{1+\tau \lambda_{S}}\right. \\
& -\frac{\tau \lambda_{S}}{1+\tau \lambda_{S}+(1-\tau) \lambda_{M}} \\
& \left.. \frac{a+\alpha q-\beta\left(v_{M}+v_{S}+\varepsilon q\right)}{\beta}\right] \times[a+\alpha q \\
& \left.-\beta\left(w+v_{M}\right)-\frac{\beta(1-\tau) \lambda_{M}\left(w-v_{S}-\varepsilon q\right)}{1+\tau \lambda_{S}}\right] \\
& -\frac{\left(1+\lambda_{M}-\tau \lambda_{M}\right)\left(1+\lambda_{S}\right) c q^{2}}{1+\tau \lambda_{S}+(1-\tau) \lambda_{M}} .
\end{aligned}
$$

Maximizing (16) gives the supplier's optimal decisions and further the equilibrium strategies for both members, which are shown in Theorem 3.

Theorem 3. In a decentralized supply chain where both the supplier and manufacturer are fairness-concerned, there exists a unique equilibrium solution for both members, which is specifically as follows:

(i) When $T<0$, then $q^{F *}=0, w^{F *}=v_{S}+\kappa\left[a-\beta\left(v_{M}+\right.\right.$ $\left.\left.v_{S}\right)\right] / \beta \xi, p^{F *}=v_{M}+v_{S}+\psi\left[a-\beta\left(v_{M}+v_{S}\right)\right] / 2 \beta \xi$.

(ii) When $T \geq 0$, then $q^{F *}=T\left[a-\beta\left(v_{M}+v_{S}\right)\right] /\left(4 \beta c \xi-T^{2}\right)$, $w^{F *}=v_{S}+(4 c \kappa+\varepsilon T)\left[a-\beta\left(v_{M}+v_{S}\right)\right] /\left(4 \beta c \xi-T^{2}\right), p^{F *}=$ $v_{M}+v_{S}+(2 c \psi+\varepsilon T)\left[a-\beta\left(v_{M}+v_{S}\right)\right] /\left(4 \beta c \xi-T^{2}\right)$, where $\xi=\left(2+\tau \lambda_{S}\right)\left(1+\lambda_{M}-\tau \lambda_{M}\right), \psi=\left(3+2 \tau \lambda_{S}\right)\left(1+\lambda_{M}-\tau \lambda_{M}\right)$, and $\kappa=\left(1+\tau \lambda_{S}\right)^{2}\left(1+\lambda_{M}-\tau \lambda_{M}\right) /\left(1+\tau \lambda_{S}+(1-\tau) \lambda_{M}\right)$.

Proof. See Appendix.

From Theorem 3, we can easily derive Corollary 4 for the special case where $\lambda_{S}=\lambda_{M}=0$.

Corollary 4. In a decentralized supply chain where both the supplier and manufacturer are fairness-neutral, a unique equilibrium solution for both members can be specifically given as follows:

(i) When $T<0$, then $q^{N *}=0, w^{N *}=\left(a-\beta\left(v_{M}-v_{S}\right)\right) / 2 \beta$, $p^{N *}=\left(3 a+\beta\left(v_{M}+v_{S}\right)\right) / 4 \beta$. (ii) When $T \geq 0$, then $q^{N *}=T\left[a-\beta\left(v_{M}+v_{S}\right)\right] /\left(8 \beta c-T^{2}\right)$, $w^{N *}=v_{S}+(4 c+\varepsilon T)\left[a-\beta\left(v_{M}+v_{S}\right)\right] /\left(8 \beta c-T^{2}\right), p^{N *}=$ $v_{M}+v_{S}+(6 c+\varepsilon T)\left[a-\beta\left(v_{M}+v_{S}\right)\right] /\left(8 \beta c-T^{2}\right)$.

Substituting the above equilibrium solutions in Theorem 3 and Corollary 4 backwards into (1), (10), and (11), the demand of the end market and profit functions of both members and the whole channel at the equilibria are presented in Tables 1 and 2.

\section{Comparisons and Sensitivity Analysis}

So far, we have derived the expressions for both members' equilibrium strategies and corresponding payoffs under different decision structures. In this section, we place emphasis on comparing these equilibrium values and discussing the impacts of some key parameters (including $\lambda_{M}, \lambda_{S}$, and $\tau$ ) on them.

To better explore some implication, we define $H:=T^{2} / \beta c$ $=(\alpha-\beta \varepsilon)^{2} / \beta c$ in a quality-sensitive market (i.e., $\left.T \geq 0\right)$. Similar to Wang et al. [19], $H$ indicates the market desire to innovate to some extent. A large value of $H$ denotes the fact that the market has a relatively great desire to innovate to improve product quality, that is, the higher the innovation-desirability-index, the greater the market desire to innovate. Therefore, $H$ can be called the market innovationdesirability-index. The condition of (9) indicates that the reasonable range of $H$ is $0<H<4$.

5.1. Analysis on the Equilibrium Quality Levels. The properties of the equilibrium quality levels are given in Property 5.

Property 5. For any $T \geqslant 0$,

(i) $q^{C *}>2 q^{N *}, q^{N *}>q^{F *}, d\left(q^{C *} / q^{N *}\right) / d H>0$, and $d\left(q^{N *} / q^{F *}\right) / d H>0$.

(ii) $\partial\left(q^{F *}\right) / \partial \lambda_{S}<0, \partial\left(q^{F *}\right) / \partial \lambda_{M}<0$.

(iii) If $\lambda_{S}<2 \lambda_{M} /\left(1+\lambda_{M}\right)$, then $\partial\left(q^{F *}\right) / \partial \tau>0$; if $\lambda_{M}<$ $\lambda_{S} /\left(2+\lambda_{S}\right)$, then $\partial\left(q^{F *}\right) / \partial \tau<0$; otherwise, $\partial\left(q^{F *}\right) / \partial \tau<0$ for $\tau<\tau_{q}$, and $\partial\left(q^{F *}\right) / \partial \tau>0$ for $\tau>\tau_{q}$, where $\tau_{q}=\left(\lambda_{S}+\right.$ $\left.\lambda_{S} \lambda_{M}-2 \lambda_{M}\right) / 2 \lambda_{S} \lambda_{M}$.

Proof. See Appendix.

Property 5(i) and (ii) gives us the following observations for a quality-sensitive market. (1) A supply chain under integrated decision-making always provides products of higher quality than that under decentralized decision-making; the product quality in an integrated supply chain is at least twice as much as that in its decentralized counterpart. And the difference in product quality between the integrated and decentralized channels increases with $H$. That is, the higher the market desire to innovate is, the greater the difference in product quality is. (2) For a decentralized supply chain, the more fairness-concerned either the supplier or the manufacturer is, the lower product quality will be selected. Hence, products provided by a fairness-concerned channel have even lower quality than those by its corresponding fairness-neutral channel. Additionally, the higher the market 
desire to innovate is, the greater the difference in product quality between the fairness-neutral channel and its fairnessconcerned counterpart is. (3) Therefore, the integrated setting where both members cooperate as a whole has an advantage in improving product quality over the decentralized setting. And the members' preference of fairness concern and facing a market with higher desire to innovate will make this advantage more apparent.

Property 5(iii) reveals that for the fairness-concerned channel facing a quality-sensitive market, the impact of bargaining power on the equilibrium quality under decentralized setting depends on the quantitative relationship of fairness concern coefficients between both members. Specifically, when the supplier's strength of fairness concern is small enough compared to the manufacturer's (see region (I) in Figure 1), the equilibrium product quality increases with his bargaining power $\tau$. However, when the supplier's strength of fairness concern is large enough compared to the manufacturer's (see region (II) in Figure 1), the equilibrium quality decreases with his bargaining power $\tau$. Besides, if both members' strengths of fairness concern are relatively close (see region (III) in Figure 1), the equilibrium quality initially decreases and then increases with the supplier's bargaining power $\tau$.

\subsection{Analysis on the Equilibrium Pricing}

\subsubsection{Effects of Fairness Concern and Bargaining Power on the Equilibrium Wholesale Price}

Property 6. (i) $\partial\left(w^{F *}\right) / \partial \lambda_{M}<0$.

(ii) When $T<0, \partial\left(w^{F *}\right) / \partial \lambda_{S}>0$ and $\partial\left(w^{F *}\right) / \partial \tau>0$.

(iii) When $T \geq 0$, (a) for the case of $\lambda_{M}=0$, if $\alpha T \geq 4 \beta c>$ $T^{2}$, then $\partial\left(w^{F *}\right) / \partial \lambda_{S} \leqslant 0, \partial\left(w^{F *}\right) / \partial \tau \leqslant 0$; if $4 \beta c>\alpha T$, then $\partial\left(w^{F *}\right) / \partial \lambda_{S}>0, \partial\left(w^{F *}\right) / \partial \tau>0$; (b) for the case of $\lambda_{S}=0$, $\partial\left(w^{F *}\right) / \partial \tau>0$.

Proof. It follows directly from the partial derivative expressions.

Property 6(i) and (ii) shows that in a price-sensitive market, the supplier's equilibrium wholesale price decreases with the manufacturer's strength of fairness concern, but increases with his own strength of fairness concern and bargaining power. These make sense because when the manufacturer's fairness concern and bargaining power decrease or the supplier's fairness concern increases, the supplier's relative power will increase, which directly leads to the increase of his wholesale price. This explanation is valid especially for the price-sensitive market where the equilibrium quality level constantly stays at zero.

Property 6(i) also indicates that in a quality-sensitive market, the equilibrium wholesale price still decreases with the manufacturer's strength of fairness concern. This can be explained from two aspects. First, similar to the case of pricesensitive market, the increase of the manufacturer's fairness concern means she is more aggressive and the supplier's relative power decreases, which in turn results in the decrease of the wholesale price for a given product quality. Second,

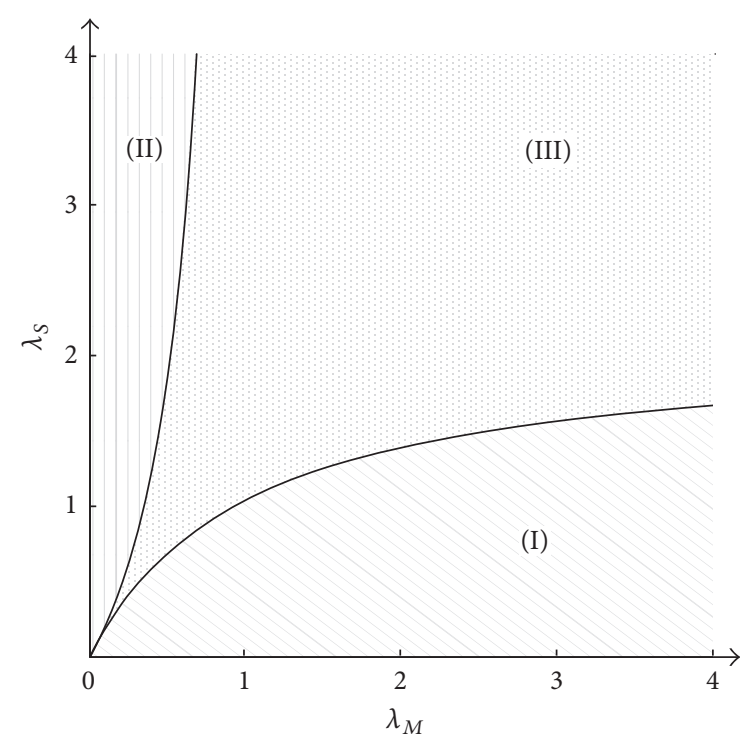

FIgURE 1: Different regions of $\lambda_{S}$ and $\lambda_{M}$ affecting the impact of $\tau$ on $q^{F *}$.

From Property 5, the increase in the manufacturer's fairness concern leads to the decrease in the equilibrium product quality, which further causes the supplier to correspondingly take a lower wholesale price.

The above two aspects of reasons can also be used to understand the impacts of changes in the supplier's strength of fairness concern and bargaining power on the equilibrium wholesale price in a quality-sensitive market. Specifically, the increase of the supplier's fairness concern and bargaining power will bring about the increase of the wholesale price for a given product quality. Meanwhile, either the increase of the supplier's fairness concern or the increase of his bargaining power when $\lambda_{M}$ is sufficiently smaller than $\lambda_{S}$ will lead to the decrease of product quality (see Property 5), and then a correspondingly lower wholesale price is requested. Property 6(iii) indicates that when $\lambda_{M}$ tends to 0 , only within a certain range of relatively small $c$ (i.e., $\alpha T / 4 \beta \geqslant c>$ $T^{2} / 4 \beta$ ) does the latter negative effect dominate the former positive one, resulting in the decrease in the wholesale price; otherwise, the former positive effect dominates the latter negative one and leads to the increase in the wholesale price. It is also easy to see that when $\lambda_{S}$ tends to 0 , the wholesale price increases as the supplier's bargaining power increases because both the two effects are positive at this time.

We wonder whether the insights from Property 6(iii) are kept for cases where the values of $\lambda_{M}$ and $\lambda_{S}$ are more general. To answer this problem, the following numerical analyses are conducted. Figures 2 and 3 display graphs plotting the impacts of $\lambda_{S}$ and $\tau$ on the equilibrium wholesale price for different values of $c$ and $\lambda_{M}$ and specific values for the other parameters in a quality-sensitive market. It is verified again that only when both $c$ and $\lambda_{M}$ are sufficiently small may the equilibrium wholesale price decrease, or decrease initially and then increase with $\lambda_{S}$ and $\tau$. Otherwise, in most cases, it will increase with $\lambda_{S}$ and $\tau$. 

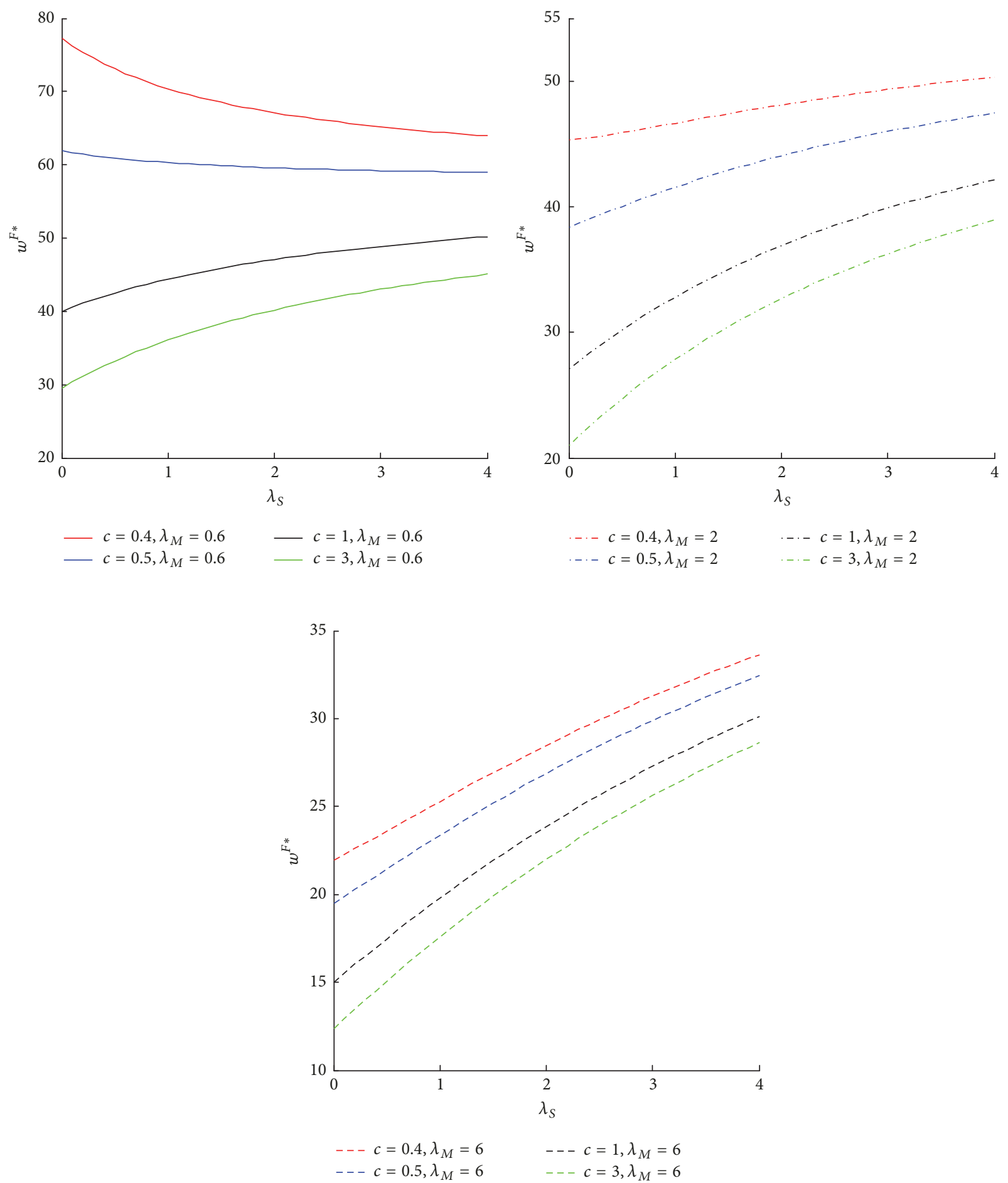

FIGURE 2: Effects of $\lambda_{S}$ on the equilibrium wholesale price $\left(a=600, \alpha=8, \beta=10, v_{S}=3, v_{M}=2, \tau=0.6, \varepsilon=0.4\right)$.

\subsubsection{Analysis on the Manufacturer's Equilibrium Selling Price}

\section{Property 7.}

(i) When $T<0, p^{C *}<p^{N *}<p^{F *}, \partial\left(p^{F *}\right) / \partial \lambda_{M}=0$, $\partial\left(p^{F *}\right) / \partial \lambda_{S}>0$, and $\partial\left(p^{F *}\right) / \partial \tau>0$.

(ii) When $T \geqslant 0$, (a) if $2 \beta c<\alpha T$, then $p^{F *}<p^{N *}<p^{C *}$; if $\alpha T<$ $2 \beta c<\alpha T+\Delta_{1}$, then $p^{F *}<p^{C *}<p^{N *}$; if $\alpha T+$ $\Delta_{1}<2 \beta c<\alpha T+\Delta_{2}$, then $p^{C *}<p^{F *}<p^{N *}$; if $2 \beta c>\alpha T+\Delta_{2}$, then $p^{C *}<p^{N *}<p^{F *}$, where $\Delta_{1}=\left((\alpha+\beta \varepsilon) /\left(1+\tau \lambda_{S}\right)\right)\left(T(1-\tau) \lambda_{M} / 2[1+(1-\right.$ $\left.\left.\tau) \lambda_{M}\right]\right), \Delta_{2}=\left((3 \alpha+\beta \varepsilon) / \tau \lambda_{S}\right)\left(T(1-\tau) \lambda_{M} / 2[1+\right.$ $\left.\left.(1-\tau) \lambda_{M}\right]\right)$; 

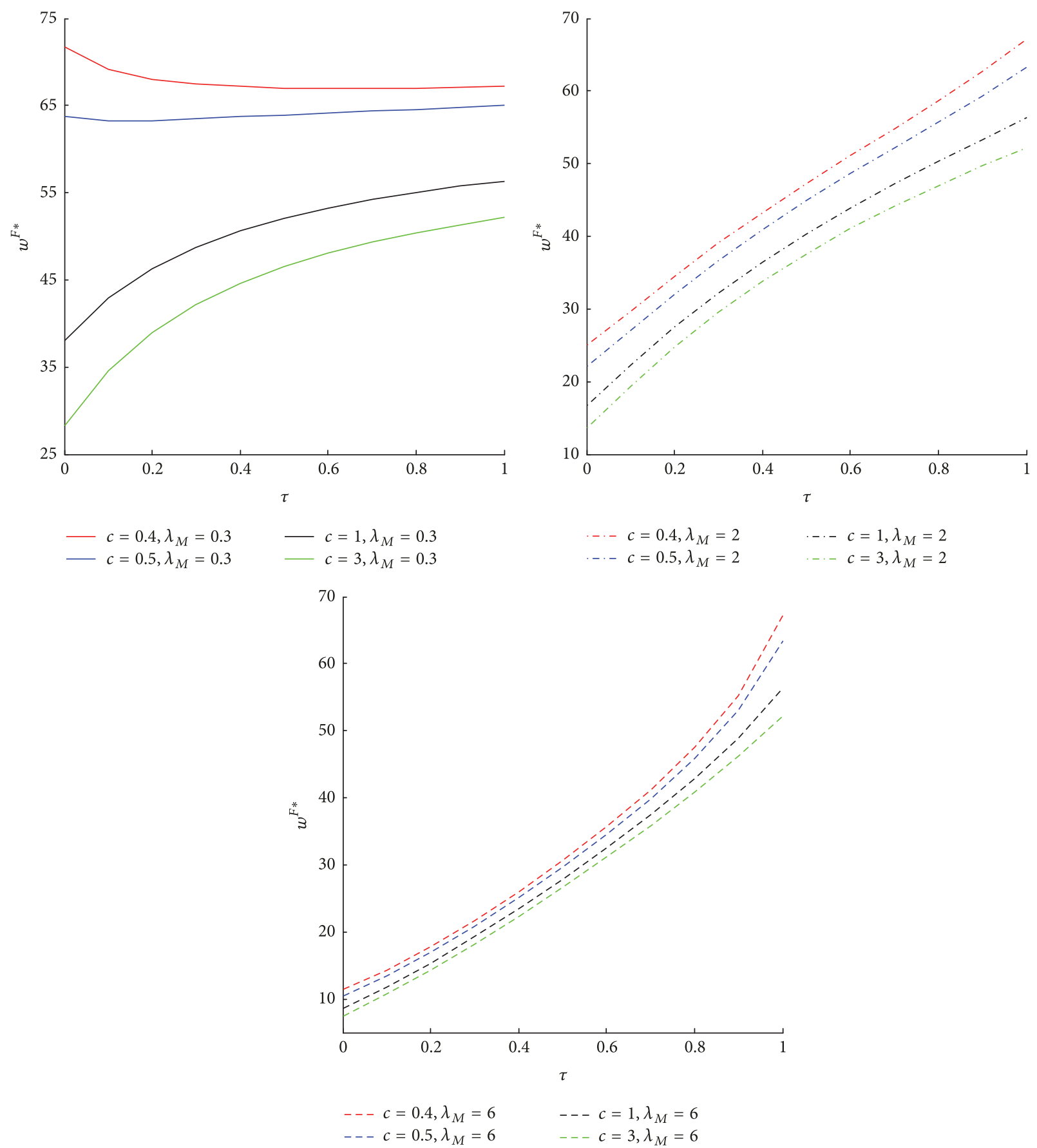

FIgURE 3: Effects of $\tau$ on the equilibrium wholesale price $\left(a=600, \alpha=8, \beta=10, v_{S}=3, v_{M}=2, \lambda_{S}=5, \varepsilon=0.4\right)$.

(b) $\partial\left(p^{F *}\right) / \partial \lambda_{M}<0$;

(c) $\partial\left(p^{F *}\right) / \partial \lambda_{S}<0$ if $\alpha T>2 \beta c\left[1+(1-\tau) \lambda_{M}\right]$, and $\partial\left(p^{F *}\right) / \partial \lambda_{S} \geqslant 0$ otherwise.

Proof. It follows directly from the expressions for the partial derivatives and the differences among $p^{F *}, p^{N *}$, and $p^{C *}$.

Several insights can be inferred from Property 7. First, Property 7(i) states that, in a price-sensitive market, the manufacturer's selling price under decentralized setting is higher than its counterpart under integrated setting, and the former increases with the supplier's strength of fairness concern and his bargaining power, whereas it is independent of the manufacturer's fairness concern. This is a phenomenon known as "double marginalization," which widely exists in traditional quality-irrelevant channels. In fact, the supplier in a price-sensitive market is always unwilling to improve product quality; hence the channel we studied here is reduced to be quality-irrelevant. We can also find that the double marginalization effect at this time will be magnified as the 
supplier is more fairness-concerned or has more bargaining power.

Second, Property 7(ii-a) shows that in a quality-sensitive market, four different order relations among $p^{F *}, p^{N *}$, and $p^{C *}$ correspond to different ranges of the supplier's qualityrelated fixed cost coefficient $c$. Specifically, when $c$ is very small such that $c \in\left(T^{2} / 4 \beta, \alpha T / 2 \beta\right]$, the equilibrium selling price under decentralized setting is lower than that under integrated setting, and the members' fairness concerns make the decentralized channel set a further lower selling price. As $c$ gets large enough such that $c>\alpha T / 2 \beta$, if both members are fairness-neutral, the selling price under decentralized setting starts to surpass that under integrated setting. However, as for a fairness-concerned channel, its selling price under decentralized setting is initially still lower than its counterpart under integrated setting till $c \geqslant\left(\alpha T+\Delta_{1}\right) / 2 \beta$. And when $c$ is sufficiently large such that $c>\left(\alpha T+\Delta_{2}\right) / 2 \beta$, under the decentralized setting alone, the selling price of a fairnessconcerned channel is higher than its corresponding fairnessneutral channel, which is the same as in a price-sensitive market. Figure 4 illustrates the different cases in Property 7(iia). Particularly, if the manufacturer is fairness-neutral, that is, $\lambda_{M}=0$, then $\Delta_{1}=\Delta_{2}=0$ and therefore the middle two situations in Figure 4 no longer exist; if the supplier is fairness-neutral, that is, $\lambda_{S}=0$, then $\Delta_{2} \rightarrow+\infty$ and hence the rightmost situation in Figure 4 will vanish.

As shown in Property 7 and Figure 4, if $c \in\left(T^{2} / 4 \beta,(\alpha T+\right.$ $\left.\left.\Delta_{1}\right) / 2 \beta\right]$ in a quality-sensitive market, then $p^{F *} \leqslant p^{C *}$. An accompanying question is whether the double marginalization effect is alleviated by the members' fairness concerns in this case. Actually, in a quality-sensitive market the double marginalization effect is also always magnified by fairness concerns. This conclusion can be got from the analysis on the channel's equilibrium total profit in Section 5.3. The underlying reason why different quantitative relations among $p^{F *}, p^{N *}$, and $p^{C *}$ occur in a quality-sensitive market is that the products have different quality levels for different scenarios, while the quality level of products in a price-sensitive market keeps constant.

Third, Property 7 (ii-b) tells us that, unlike in a pricesensitive market, the manufacturer's equilibrium selling price under decentralized setting in a quality-sensitive market decreases with her strength of fairness concern. That is, the more fairness-concerned the manufacturer is, the lower the selling price will be set. This coincides with intuitive reasoning because both the product quality and wholesale price imposed by the supplier decrease as the manufacturer gets more fairness-concerned.

However, Property 7(ii-c) indicates that, in a qualitysensitive market, the effect of the supplier's fairness concern on the selling price under decentralized setting is uncertain. When the quality-related fixed cost coefficient $c$, the manufacturer's fairness concern coefficient $\lambda_{M}$, and her bargaining power $(1-\tau)$ are sufficiently small such that $c[1+(1-$ $\left.\tau) \lambda_{M}\right]<\alpha T / 2 \beta$, the selling price decreases as the supplier becomes more fairness-concerned; otherwise, it increases in the supplier's strength of fairness concern. Again in a quality-sensitive market, a similar effect of the supplier's

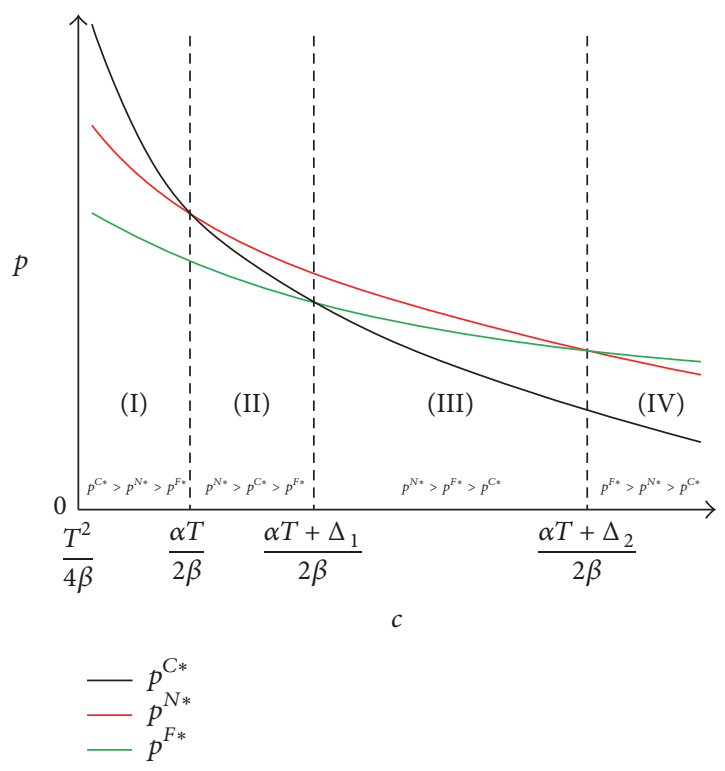

FIGURE 4: Different quantitative relations among $p^{F *}, p^{N *}$, and $p^{C *}$ in a quality-sensitive market.

bargaining power $\tau$ on the selling price under decentralized setting can be observed from numerical analyses in Figure 5. Only when $\lambda_{M}$ is sufficiently small compared to $\lambda_{S}$ and $c$ is small enough too, might the selling price $p^{F *}$ decreases as the supplier gets more bargaining power (See the upper two lines in Figure 5(a)). However, in other most cases, $p^{F *}$ increases with $\tau$. The effects of $\lambda_{S}$ and $\tau$ on $p^{F *}$ are very similar to those on $w^{F *}$, which is probably because the manufacturer wants to keep her margin stable to some extent.

In addition, by comparing Properties 7 and 2, one can find that the effects of $\lambda_{M}, \lambda_{S}$, and $\tau$ on the equilibrium selling price are quite different from the effects of them on the manufacturer's best pricing response to any given $q$ and $w$. Meantime, Xie et al. [21] showed that if the wholesale price is exogenously given, supply chains adopting vertical integration strategy tend to provide products with "higher quality, higher selling price" compared with those adopting decentralization strategies. While in our model, where the wholesale price is endogenous, it is found that vertical integration strategy still leads to higher quality, but it might lead to lower selling price in most cases.

\subsection{Analysis on the Equilibrium Demand and Profits}

Property 8. (i) $D^{C *} \geqslant 2 D^{N *} \geqslant 2 D^{F *}$; when $T \geqslant 0, d\left(D^{C *} /\right.$ $\left.D^{N *}\right) / d H>0$ and $d\left(D^{N *} / D^{F *}\right) / d H>0$.

(ii) $\partial\left(D^{F *}\right) / \partial \lambda_{S}<0$ always holds; when $T \geqslant 0, \partial\left(D^{F *}\right) /$ $\partial \lambda_{M}<0$; otherwise $\partial\left(D^{F *}\right) / \partial \lambda_{M}=0$.

(iii) (a) When $T<0, \partial\left(D^{F *}\right) / \partial \tau<0$; (b) when $T \geqslant 0$, $\partial\left(D^{F *}\right) / \partial \tau>0$ if $H>4 \lambda_{S}\left(1+\lambda_{M}\right)^{2} / \lambda_{M} ; \partial\left(D^{F *}\right) / \partial \tau<0$ if $H<4 \lambda_{S} / \lambda_{M}$; Otherwise, $\partial\left(D^{F *}\right) / \partial \tau<0$ for $\tau<\tau_{D}$, and $\partial\left(D^{F *}\right) / \partial \tau>0$ for $\tau>\tau_{D}$, where $\tau_{D}=1+\left(1 / \lambda_{M}\right)(1-$ $\left.\sqrt{H \lambda_{M} / 4 \lambda_{S}}\right)$. 


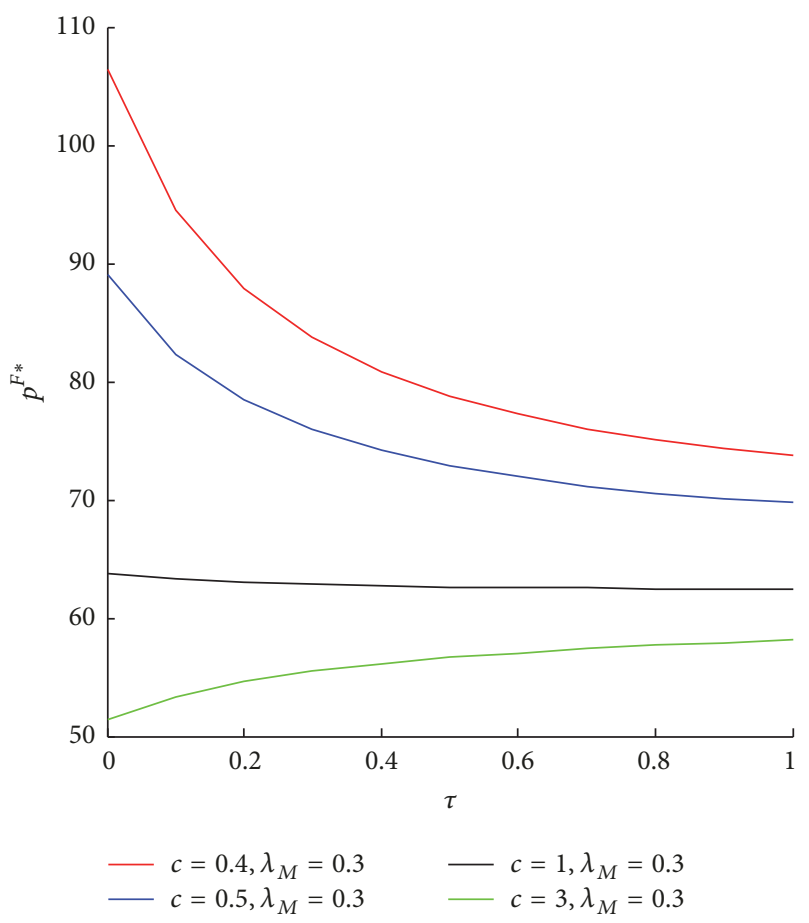

(a)

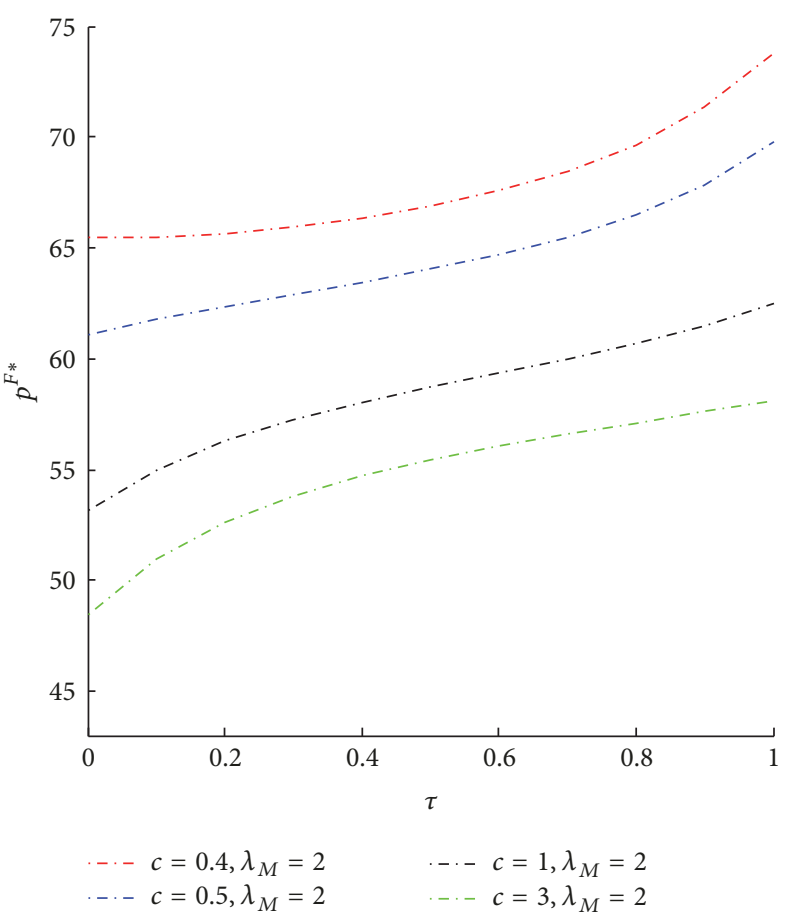

(b)

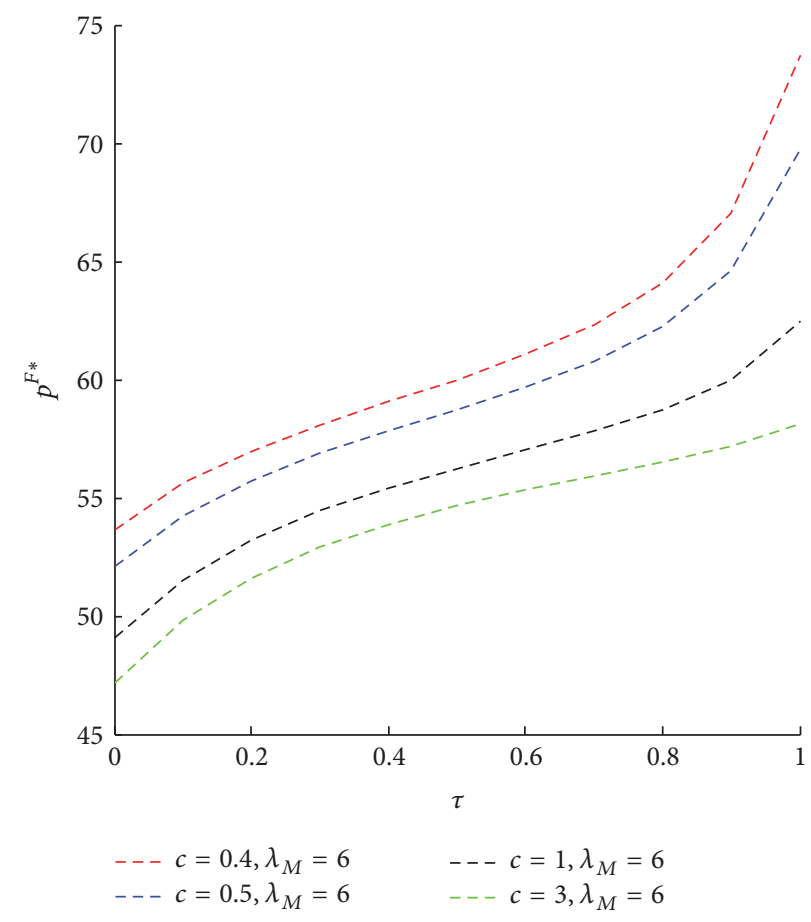

(c)

FIGURE 5: Effects of $\tau$ on the equilibrium selling price $\left(a=600, \alpha=8, \beta=10, v_{S}=3, v_{M}=2, \lambda_{S}=5, \varepsilon=0.4\right)$.

Proof. See Appendix.

The following insights on the equilibrium demand can be obtained from Property 8 . (1) The equilibrium demand under integrated setting is at least twice that under decentralized setting. And in a quality-sensitive market, the difference between them increases with $H$. That is, the higher the market desire to innovate is, the greater the integrated channel's advantage in generating demand will be. This again complements Xie et al. [21], who reported that supply chains 
adopting decentralization strategies lead to more demand than adopting vertical integration strategy when the wholesale price is exogenous.

(2) In a decentralized fairness-concerned channel, the equilibrium demand $D^{F *}$ always decreases with the supplier's fairness concern no matter what type the market is. It also decreases with the manufacturer's fairness concern in a quality-sensitive market, but it is independent of the manufacturer's fairness concern in a price-sensitive market. Therefore, the equilibrium demand of a decentralized fairness-concerned channel is necessarily not more than that of its corresponding decentralized fairness-neutral channel. Besides, in a quality-sensitive market, the fairnessneutral channel's advantage in generating demand over the fairness-concerned channel is also positively correlated with $H$.

(3) In a price-sensitive market, the equilibrium demand of a decentralized fairness-concerned channel $D^{F *}$ always decreases with the supplier's bargaining power $\tau$. This is because a lower selling price will be set while the product quality keeps constant as $\tau$ increases. But how about a qualitysensitive market? In such a market, Property 8(iii-b) states that the impact of $\tau$ on $D^{F *}$ is uncertain and closely related to $H$ and both members' fairness concern. Particularly, $D^{F *}$ will always increase with $\tau$ if the market desire to innovate is higher than a particular threshold which correlates with $\lambda_{M}$ and positively with $\lambda_{S}$. This implies that, in a quality-sensitive market, when the market desire to innovate is sufficiently high and the supplier's strength of fairness concern is far smaller than the manufacturer's, more bargaining power of the supplier will be beneficial to stimulate demand.

Property 9. (i) $\pi_{\mathrm{SC}}^{C *} \geq(4 / 3) \pi_{\mathrm{SC}}^{N *}, \pi_{\mathrm{SC}}^{N *} \geq \pi_{\mathrm{SC}}^{F *}$; when $T \geqslant 0$, $d\left(\pi_{\mathrm{SC}}^{\mathrm{C} *} / \pi_{\mathrm{SC}}^{N *}\right) / d H>0, d\left(\pi_{\mathrm{SC}}^{N *} / \pi_{\mathrm{SC}}^{F *}\right) / d H>0$.

(ii) $\partial\left(\pi_{\mathrm{SC}}^{F *}\right) / \partial \lambda_{S}<0$; when $T \geqslant 0, \partial\left(\pi_{\mathrm{SC}}^{F *}\right) / \partial \lambda_{M}<0$; otherwise $\partial\left(\pi_{\mathrm{SC}}^{F *}\right) / \partial \lambda_{M}=0$.

(iii) (a) When $T<0, \partial\left(\pi_{\mathrm{SC}}^{F *}\right) / \partial \tau<0$; (b) when $T \geqslant 0$, for the case of $\lambda_{M} \rightarrow 0$ or $H \rightarrow 0, \partial\left(\pi_{\mathrm{SC}}^{F *}\right) / \partial \tau<0$, while for the case of $\lambda_{S} \rightarrow 0, \partial\left(\pi_{\mathrm{SC}}^{F *}\right) / \partial \tau>0$.

Proof. See Appendix.

Property 9 shows that the impacts of $\lambda_{M}, \lambda_{S}$, and $\tau$ on the overall profit of the supply chain are similar to those on the demand. First, the overall profit under integrated setting is always greater than that under decentralized settings, and the former is at least one-third more than the latter. This is consistent with our intuition. Decisions under integrated setting are made to maximize the whole channel's performance, while under decentralized setting, each member optimizes his/her own goal independently. Second, in a decentralized supply chain, the system's overall profit decreases with both members' strengths of fairness concern, except that when $T<0$ it is irrelevant to the manufacturer's fairness concern. Hence, the profit of a decentralized fairness-concerned channel will be no more than its corresponding fairness-neutral channel. And both the ratios of $\pi_{\mathrm{SC}}^{C *}$ to $\pi_{\mathrm{SC}}^{N *}$ and of $\pi_{\mathrm{SC}}^{N *}$ to $\pi_{\mathrm{SC}}^{F *}$ also increase with $H$. The above two insights confirm that a decentralized supply chain is worse off than its vertically integrated counterpart due to double marginalization no matter whether the members are fairness-concerned or not. The members' fairness concern even makes the decentralized supply chain much worse off. Third, in a price-sensitive market, the overall profit of a decentralized fairness-concerned channel is always decreasing in the supplier's bargaining power, while in a quality-sensitive market, it is more likely to increase (decrease) as the supplier's bargaining power increases when the supplier (manufacturer) tends to be fairness-neutral and when the market desire to innovate is sufficiently high (very low and tends to zero).

Property 10 further investigates the impacts of $\lambda_{M}, \lambda_{S}$, and $\tau$ on the profit of each member in a decentralized supply chain.

Property 10. (i) (a) $\partial\left(\pi_{M}^{F *}\right) / \partial \lambda_{S}<0$; (b) when $T<0, \partial\left(\pi_{S}^{F *}\right) /$ $\partial \lambda_{S}>0$ if and only if $\tau \lambda_{S}\left(1+\tau \lambda_{S}\right) /(1-\tau) \lambda_{M}<2$; when $T \geq 0$, if $\tau \lambda_{S}\left(1+\tau \lambda_{S}\right) /(1-\tau) \lambda_{M}>2$, then $\partial\left(\pi_{S}^{F *}\right) / \partial \lambda_{S}<0$; otherwise the sign of $\partial\left(\pi_{S}^{F *}\right) / \partial \lambda_{S}$ is uncertain.

(ii) (a) $\partial\left(\pi_{S}^{F *}\right) / \partial \lambda_{M}<0$; (b) when $T<0, \partial\left(\pi_{M}^{F *}\right) / \partial \lambda_{M}>$ 0 , while when $T \geq 0, \partial\left(\pi_{M}^{F *}\right) / \partial \lambda_{M}>0$ if and only if $H<$ $4 \xi_{\kappa} /(\psi+\delta-\kappa)$, where $\delta=\tau \lambda_{S}\left[1+2\left(1+\tau \lambda_{S}\right)(1-\tau) \lambda_{M} /(1+\right.$ $\left.\left.\tau \lambda_{S}+(1-\tau) \lambda_{M}\right)\right]$

(iii) (a) when $T<0, \partial\left(\pi_{M}^{F *}\right) / \partial \tau<0$ always holds, but $\partial\left(\pi_{S}^{F *}\right) / \partial \tau>0$ if and only if $\lambda_{M}>\tau \lambda_{S}{ }^{2}\left(1+\tau \lambda_{S}\right) /(2(1+$ $\left.\lambda_{S}\right)+\tau \lambda_{S}\left(1+\tau \lambda_{S}\right)$ ); (b) when $T \geq 0$, for the case of $\lambda_{S} \rightarrow 0$, $\partial\left(\pi_{S}^{F *}\right) / \partial \tau>0$ always holds, but $\partial\left(\pi_{M}^{F *}\right) / \partial \tau>0$ if and only if $H>\left(8+8(1-\tau) \lambda_{m}\right) /\left(2+3(1-\tau) \lambda_{m}\right)$; and for the case of $\lambda_{M} \rightarrow 0, \partial\left(\pi_{M}^{F *}\right) / \partial \tau<0$ and $\partial\left(\pi_{S}^{F *}\right) / \partial \tau<0$ always hold.

Proof. See Appendix.

From Property 10(i) and (ii), we can find that in a decentralized supply chain, one member's fairness concern will make the other member's profitability worse off. And the more fairness-concerned one member is, the greater the decrease in the other member's profit will be. This can be partly explained by the fact that, analogous to Du et al. [32], a fairness-concerned member in our study means he/she has a dominant preference called status-seeking, besides the preference of mutual care and empathy for others. Then another question of interest is what happens to the member's own profit as he/she gets more fairness-concerned. Property 10(i) and (ii) also reveals that the impact of the member's fairness concern on his/her own profit is uncertain. Property 10(i-b) indicates that only when the product of the manufacturer's fairness concern parameter $\lambda_{M}$ and her bargaining power $(1-\tau)$ are sufficiently great such that $2(1-\tau) \lambda_{M}>\tau \lambda_{S}(1+$ $\left.\tau \lambda_{S}\right)$, it is possible that the supplier's profit increases as he is more fairness-concerned; otherwise, it always decreases. Property 10(ii-b) states that in a price-sensitive market, the manufacturer's profit always increases with her own fairness concern parameter $\lambda_{M}$, while in a quality-sensitive market, it increases with $\lambda_{M}$ only if the market desire to innovate is sufficiently low. A direct insight from this observation is that a fairness-concerned channel member may be willing 
to sacrifice some of his/her own monetary payoff to lower the other member's payoff and move into a more equitable outcome. This is also consistent with the past studies on fairness concern (see Charness and Rabin [39] and Cui et al. [24]).

Property 10(iii-a) gives the effects of members' bargaining power on their profitability in the decentralized fairnessconcerned channel in a price-sensitive market. The manufacturer's profit always decreases as the supplier has more bargaining power, while the supplier's profit increases with his own bargaining power $\tau$ only if the manufacturer's fairness concern parameter $\lambda_{M}$ is greater than a certain threshold, which is positively associated with the supplier's fairness concern parameter $\lambda_{S}$ and bargaining power $\tau$. In other words, the supplier's profit increases with $\tau$ only when his strength of fairness concern is sufficiently smaller than the manufacturer's and when $\tau$ is in a range of relatively small values.

In a quality-sensitive market, due to the complexity, it is hard to determine the effects of $\tau$ on both members' profitability in the decentralized fairness-concerned channel. Hence, it is necessary to resort to special cases in order to develop some hints for more general cases. From Property 10(iii-b), when the supplier tends to be fairness-neutral, the more bargaining power of the supplier will make him get higher profit, while making the manufacturer also earn more if the market desire to innovation and the manufacturer's strength of fairness concern are great enough such that $H>\left(8+8(1-\tau) \lambda_{m}\right) /\left(2+3(1-\tau) \lambda_{m}\right)$. In contrast, when the manufacturer tends to be fairness-neutral, the more bargaining power of the supplier will always make both him and the manufacturer worse off.

Synthesizing the above observations about the implications of $\tau$ on channel members' profit in extreme cases can give us some insights for general cases in a qualitysensitive market. The supplier's profit increases with his own bargaining power $\tau$ only when his strength of fairness concern is far smaller than the manufacturer's; otherwise it more likely decreases with $\tau$. In addition to a price-sensitive market, the manufacturer's profit also increases with her own bargaining power $1-\tau$ (i.e., decreases with $\tau$ ) in a quality market if her strength of fairness concern is far smaller than the supplier's and the market desire to innovation is low enough; otherwise it often tends to increase with $\tau$. These can also be verified by numerical examples, which are omitted here due to space limitations. An interesting conclusion is implied: the increase of a channel member's bargaining power is neither always beneficial to him/her nor always harmful to his/her counterpart. In general, one member's sufficiently smaller strength of fairness concern than his/her counterpart's is more conducive to lead both him/her and his/her counterpart to get higher profits as his/her bargaining power increases, which in turn results in higher overall profit of the whole channel.

\section{Conclusions}

In this paper, fairness concern on the part of channel members are incorporated into the investigation on product quality and pricing decisions in a two-echelon supply chain consisting of a supplier and a manufacturer. The supplier invests in the technology to improve quality and provides core components with a wholesale price contract, which are processed by the manufacturer into final products to be sold to consumers. Quality level, wholesale price of core components, and selling price of final products are set by the supplier and the manufacturer, respectively. Both the supplier and the manufacturer are assumed to be fairness-concerned, by using Nash bargaining solutions as their fairness reference points. The models of vertically integrated supply chain as well as decentralized one are formulated to determine the equilibrium product quality and pricing strategies of the dyadic supply chain with members' fairness preference.

This study makes several significant contributions to our understanding of supply chain management. First, in contrast to previous studies, almost all of them studied product quality and pricing issues and fairness concerns of decision-makers independently, whereas we address quality and pricing decisions in supply chains simultaneously considering members' preference of fairness concern; therefore, the problem is more complex but even closer to the actual practice. Second, besides both members' fairness preference, their power and contribution are endogenously considered by adopting Nash bargaining solution as their fairness reference points, which is a new approach to measure fairness concern. Based on this, we investigate the combined effects of both members' fairness concern and bargaining power on their product quality and pricing decisions and corresponding payoffs. The results we obtain show that the two members' equilibrium product quality and pricing strategies are significantly influenced by their fairness-concerned behavior, bargaining power, and market type (i.e., quality-sensitive market or price-sensitive market). An interesting observation is in a quality-sensitive market; one member's sufficiently smaller strength of fairness concern than his/her counterpart's is more conducive to lead both him/her and his/her counterpart to get higher profits as his/her bargaining power increases, which in turn results in higher overall profit of the supply chain. This means that the increase of a channel member's bargaining power is not always harmful to his/her counterpart; its impact will also be conditional on their fairness preference.

Our study has several limitations, which provide interesting directions for future research. First of all, this paper only considers a simply two-echelon supply chain where members have distributional fairness concern. It might be interesting to extend our study to the cases of multiple suppliers or multiple manufacturers with coexistence of distributional and peer-induced fairness preferences. In addition, the proposed models are developed under deterministic demand and in absence of market risk. Therefore, another future research can also consider the stochastic demand and members' risk preference (see, e.g., Zhou et al. [40]) besides fairness preference. Finally, it is implicitly assumed in our paper that consumers are homogenous and fairness-neutral for simplicity. Simultaneously incorporating consumers' fairness preference and their heterogeneity on quality into the present models is also worthy of future study. 


\section{Appendix}

Proof of Theorem 1. Apparently, under the assumption of (9), the Hessian matrix $H^{C}$ is negative definite; hence $\pi_{\mathrm{SC}}^{C}(p, q)$ is strictly jointly concave in $p$ and $q$. Taking the first derivative of $\pi_{\mathrm{SC}}^{C}(p, q)$ with respect to $p$ and $q$, and setting them to zero, we have

$$
\begin{aligned}
\frac{\partial \pi_{\mathrm{SC}}^{C}(p, q)}{\partial p}= & -2 \beta p+a+(\alpha+\beta \varepsilon) q+\beta\left(v_{M}+v_{S}\right) \\
= & 0 \\
\frac{\partial \pi_{\mathrm{SC}}^{C}(p, q)}{\partial q}= & -2(\alpha \varepsilon+c) q+(\alpha+\beta \varepsilon) p \\
& -\alpha\left(v_{M}+v_{S}\right)-\varepsilon a=0 .
\end{aligned}
$$

Solving for $p$ and $q$ simultaneously from (A.1), we obtain the unique solution as specifically expressed in part (ii) of Theorem 1. However, it is worth noting that if $T<0$, this $q^{C *}$ is negative and infeasible. Hence, in the case of $T<0$, the optimal quality level $q^{C *}$ should be zero, and the optimal selling price is $p^{C *}=\left(a+\beta\left(v_{M}+v_{S}\right)\right) / 2 \beta$, so we can get the conclusions in Theorem 1 .

Proof of Theorem 3. By taking the second derivatives of (16) with respect to $w$ and $q$, we have

$$
\begin{aligned}
& \frac{\partial^{2} u_{S}^{F}(q, w)}{\partial w^{2}} \\
& \quad=-\frac{\beta\left(1+\lambda_{S}\right)\left(2+\tau \lambda_{S}\right)\left[1+\tau \lambda_{S}+(1-\tau) \lambda_{M}\right]}{2\left(1+\tau \lambda_{S}\right)^{2}} \\
& \quad<0, \\
& \frac{\partial^{2} u_{S}^{F}(q, w)}{\partial w^{2}} \frac{\partial^{2} u_{S}^{F}(q, w)}{\partial q^{2}}-\frac{\partial^{2} u_{S}^{F}(q, w)}{\partial w \partial q} \frac{\partial^{2} u_{S}^{F}(q, w)}{\partial x \partial w} \\
& \quad=\frac{\left(1+\lambda_{S}\right)^{2}}{4\left(1+\tau \lambda_{S}\right)^{2}}\left[4 \beta c\left(2+\tau \lambda_{S}\right)\left(1+\lambda_{M}-\tau \lambda_{M}\right)\right. \\
& \left.-(\alpha-\beta \varepsilon)^{2}\right] .
\end{aligned}
$$

It is easy to see that under the assumption of (9), the right side of (A.2) is greater than zero. Hence, the associated Hessian matrix $H_{S}^{F}=\left[\begin{array}{cc}\partial^{2} u_{S}^{F}(q, w) / \partial w^{2} & \partial^{2} u_{S}^{F}(q, w) / \partial w \partial q \\ \partial^{2} u_{S}^{F}(q, w) / \partial q \partial w & \partial^{2} u_{S}^{F}(q, w) / \partial q^{2}\end{array}\right]$ is negative definitive, which means $u_{S}^{F}(q, w)$ is strictly jointly concave in $q$ and $w$, and there exists a unique equilibrium solution for $q$ and $w$.

Taking the first derivatives of (16) with respect to $q$ and $w$ and equating them to zero can give us the first-order conditions. Through solving these first-order conditions, we have

$$
q^{F *}=\frac{\left[a-\beta\left(v_{S}+v_{M}\right)\right](\alpha-\beta \varepsilon)}{4 \beta c \xi-(\alpha-\beta \varepsilon)^{2}},
$$

$$
w^{F *}=v_{S}+\frac{\left[a-\beta\left(v_{S}+v_{M}\right)\right][(\alpha-\beta \varepsilon) \varepsilon+4 c \kappa]}{4 \beta c \xi-(\alpha-\beta \varepsilon)^{2}} .
$$

Similar to the proof of Theorem 1 , it is easy to see that the equilibrium solutions in (A.3) and (A.4) are feasible only when $T \geq 0$; otherwise, the product quality given in (A.3) is negative and inappropriate.

If $T<0$, the supplier will select its optimal quality level $q^{F *}=0$. Substituting $q^{F *}=0$ into the first-order conditions and solving them give the supplier's optimal wholesale price $w^{F *}=\left(\kappa\left(a-\beta v_{M}\right)+\beta(\xi-\kappa) v_{S}\right) / \beta \xi$.

At last, substituting the supplier's optimal decision $\left(q^{F *}\right.$, $\left.w^{F *}\right)$ in the above two different cases into (14), we can obtain the manufacturer's optimal selling price as shown in Theorem 3.

Proof of Property 5. From Table 2, when $T \geq 0, q^{C *} / q^{N *}=$ $\left(8 \beta c-T^{2}\right) /\left(4 \beta c-T^{2}\right)=(8-H) /(4-H)$ and $q^{N *} / q^{F *}=$ $\left(4 \beta c \xi-T^{2}\right) /\left(8 \beta c-T^{2}\right)=(4 \xi-H) /(8-H)$. And obviously, $\xi$ is increasing both in $\lambda_{S}$ and $\lambda_{M}$, and $\xi \geq 2$, since $\lambda_{S} \geq 0$, $\lambda_{M} \geq 0$, and $0<\tau<1$. Then Property 2(i) and (ii) follow directly from the corresponding first derivative expressions and the condition of $0<H<4$.

Additionally, taking the first derivative of $\xi$ with respect to $\tau$, we have

$$
\frac{\partial \xi}{\partial \tau}=\lambda_{S}+\lambda_{S} \lambda_{M}-2 \lambda_{M}-2 \tau \lambda_{S} \lambda_{M}
$$

From (A.5) and $0<\tau<1$, it is seen that when $\lambda_{S}<$ $2 \lambda_{M} /\left(1+\lambda_{M}\right), \partial \xi / \partial \tau<0$ is always true; when $\lambda_{M}<\lambda_{S} /(2+$ $\left.\lambda_{S}\right), \partial \xi / \partial \tau>0$ is always true; otherwise, $\partial \xi / \partial \tau>0$ over the interval $0<\tau<\left(\lambda_{S}+\lambda_{S} \lambda_{M}-2 \lambda_{M}\right) / 2 \lambda_{S} \lambda_{M}$, and $\partial \xi / \partial \tau<0$ over the interval of $\left(\lambda_{S}+\lambda_{S} \lambda_{M}-2 \lambda_{M}\right) / 2 \lambda_{S} \lambda_{M}<\tau<1$. Then part (iii) of Property 2 follows.

Proof of Property 8. (i) From Tables 1 and 2, when $T<0$, $D^{C *} / D^{N *}=2$, and $D^{N *} / D^{F *}=\left(4+2 \tau \lambda_{S}\right) / 4 \geqslant 1, D^{C *}=$ $2 D^{N *} \geqslant 2 D^{F *}$. And when $T \geq 0, D^{N *} / D^{F *}=(4 \beta c \xi-$ $\left.T^{2}\right) /(2 \xi-\psi)\left(8 \beta c-T^{2}\right)=\left(4\left(2+\tau \lambda_{S}\right)\left(1+\lambda_{M}-\tau \lambda_{M}\right)-\right.$ $H) /\left(1+\lambda_{M}-\tau \lambda_{M}\right)(8-H) \geq 1$, and $D^{C *} / D^{N *}=(8 \beta c-$ $\left.T^{2}\right) /\left(4 \beta c-T^{2}\right)=(8-H) /(4-H)>2$, again $D^{C *}>2 D^{N *} \geqslant$ $2 D^{F *}$, and $d\left(D^{C *} / D^{N *}\right) / d H>0$ and $d\left(D^{N *} / D^{F *}\right) / d H>0$ follow directly from the expressions for the corresponding first derivatives using the condition of $0<H<4$.

(ii) The results can be obtained directly from the first partial derivatives of $D^{F *}$ with respect to $\lambda_{S}$ and $\lambda_{M}$; the details are omitted.

(iii) The result for the case of $T<0$ is obvious from Table 1 . In the following, we consider the case of $T \geq 0$. From Table 2

$$
\begin{aligned}
& \frac{\partial D^{F *}}{\partial \tau}=2 \beta c\left[a-\beta\left(v_{M}+v_{S}\right)\right] \\
& \cdot \frac{\left[\lambda_{M} T^{2}-4 \beta c \lambda_{S}\left(1+\lambda_{M}-\tau \lambda_{M}\right)^{2}\right]}{\left[4 \beta c\left(2+\tau \lambda_{S}\right)\left(1+\lambda_{M}-\tau \lambda_{M}\right)-T^{2}\right]^{2}}
\end{aligned}
$$




$$
\begin{aligned}
& =2\left[a-\beta\left(v_{M}+v_{S}\right)\right] \\
& =\frac{\left[\lambda_{M} H-4 \lambda_{S}\left(1+\lambda_{M}-\tau \lambda_{M}\right)^{2}\right]}{\left[4\left(2+\tau \lambda_{S}\right)\left(1+\lambda_{M}-\tau \lambda_{M}\right)-H\right]^{2}} .
\end{aligned}
$$

It is clear that the sign of (A.6) is determined by that of expression $\lambda_{M} H-4 \lambda_{S}\left(1+\lambda_{M}-\tau \lambda_{M}\right)^{2}$. Hence, $D^{F *}$ monotonically increases with $\tau \Leftrightarrow H>4 \lambda_{S}\left(1+\lambda_{M}\right.$ $\left.-\tau \lambda_{M}\right)^{2} / \lambda_{M}$. That is, when $H>4 \lambda_{S}\left(1+\lambda_{M}\right)^{2} / \lambda_{M}, \lambda_{M} H-$ $4 \lambda_{S}\left(1+\lambda_{M}-\tau \lambda_{M}\right)^{2}>0$ always holds and $\partial\left(D^{F *}\right) / \partial \tau>$ 0 ; when $H<4 \lambda_{S} / \lambda_{M}, \lambda_{M} H-4 \lambda_{S}\left(1+\lambda_{M}-\tau \lambda_{M}\right)^{2}<0$ always holds and $\partial\left(D^{F *}\right) / \partial \tau<0$; when $4 \lambda_{S} / \lambda_{M} \leq H \leq$ $4 \lambda_{S}\left(1+\lambda_{M}\right)^{2} / \lambda_{M}, \lambda_{M} H-4 \lambda_{S}\left(1+\lambda_{M}-\tau \lambda_{M}\right)^{2}<0$ if $\tau<1+$ $\left(1 / \lambda_{M}\right)\left(1-\sqrt{H \lambda_{M} / 4 \lambda_{S}}\right)$, and $\lambda_{M} H-4 \lambda_{S}\left(1+\lambda_{M}-\tau \lambda_{M}\right)^{2}>$ 0 otherwise; thus the conclusions follow.

Proof of Property 9. From Table 1, when $T<0, \pi_{\mathrm{SC}}^{C *} / \pi_{\mathrm{SC}}^{N *}=$ $4 / 3, \pi_{\mathrm{SC}}^{N *} / \pi_{\mathrm{SC}}^{F *}=3\left(2+\tau \lambda_{S}\right)^{2} / 4\left(3+2 \tau \lambda_{S}\right) \geq 1, \partial \pi_{\mathrm{SC}}^{F *} / \partial \lambda_{M}=0$, $\partial \pi_{\mathrm{SC}}^{F *} / \partial \lambda_{S}=-\tau\left(1+\tau \lambda_{S}\right)\left[a-\beta\left(v_{S}+v_{M}\right)\right]^{2} / 2 \beta\left(2+\tau \lambda_{S}\right)^{3}<0$, and $\partial \pi_{\mathrm{SC}}^{F *} / \partial \tau=-\lambda_{S}\left(1+\tau \lambda_{S}\right)\left[a-\beta\left(v_{S}+v_{M}\right)\right]^{2} / 2 \beta\left(2+\tau \lambda_{S}\right)^{3}<0$.

From Table 2 , when $T \geq 0, \pi_{\mathrm{SC}}^{C *} / \pi_{\mathrm{SC}}^{N *}=(8-H)^{2} /(4-$ $H)(12-H)$ and $\pi_{\mathrm{SC}}^{N *} / \pi_{\mathrm{SC}}^{F *}=(12-H) /(4(2 \xi-\psi) \psi-H)((4 \xi-$ $H) /(8-H))^{2}$. Based on the assumption of $0<H<4$, $d\left(\pi_{\mathrm{SC}}^{\mathrm{C} *} / \pi_{\mathrm{SC}}^{N *}\right) / d H>0$ and $d\left(\pi_{\mathrm{SC}}^{N *} / \pi_{\mathrm{SC}}^{F *}\right) / d H>0$ directly follow. Therefore, $\pi_{\mathrm{SC}}^{C *} / \pi_{\mathrm{SC}}^{N *} \geq \lim _{H \rightarrow 0} \pi_{\mathrm{SC}}^{C *} / \pi_{\mathrm{SC}}^{N *}=4 / 3, \pi_{\mathrm{SC}}^{N *} /$ $\pi_{\mathrm{SC}}^{F *} \geq \lim _{H \rightarrow 0} \pi_{\mathrm{SC}}^{N *} / \pi_{\mathrm{SC}}^{F *}=1$. Meanwhile, we can derive that

$$
\frac{\partial \pi_{\mathrm{SC}}^{F *}}{\partial \lambda_{S}}
$$

$$
\begin{gathered}
=\frac{-8 \tau\left(1+\lambda_{M}-\tau \lambda_{M}\right)\left[a-\beta\left(v_{S}+v_{M}\right)\right]^{2}}{\beta\left[4\left(2+\tau \lambda_{S}\right)\left(1+\lambda_{M}-\tau \lambda_{M}\right)-H\right]^{3}}[4(1 \\
\left.\left.+\tau \lambda_{S}\right)\left(1+\lambda_{M}-\tau \lambda_{M}\right)^{2}+(1-\tau) \lambda_{M} H\right]<0, \\
\frac{\partial \pi_{\mathrm{SC}}^{F *}}{\partial \lambda_{M}}=\frac{-8(1-\tau)\left[a-\beta\left(v_{S}+v_{M}\right)\right]^{2}}{\beta\left[4\left(2+\tau \lambda_{S}\right)\left(1+\lambda_{M}-\tau \lambda_{M}\right)-H\right]^{3}}[(1 \\
\left.\left.+\tau \lambda_{S}\right)+\left(3+2 \tau \lambda_{S}\right)(1-\tau) \lambda_{M}\right] H<0, \\
\frac{\partial \pi_{S C}^{F *}}{\partial \tau} \quad-8\left[a-\beta\left(v_{M}+v_{S}\right)\right]^{2} \\
=\frac{\beta\left[4\left(2+\tau \lambda_{S}\right)\left(1+\lambda_{M}-\tau \lambda_{M}\right)-H\right]^{3}}{\beta\left(4 \lambda_{S}(1\right.} \\
\left.+\tau \lambda_{S}\right)\left(1+\lambda_{M}-\tau \lambda_{M}\right)^{3}-\lambda_{M}\left(1+\tau \lambda_{S}\right) H+(1 \\
\left.-\tau) \lambda_{M}\left[\lambda_{S}\left(1+\lambda_{M}\right)-3 \lambda_{M}\left(1+\tau \lambda_{S}\right)\right] H\right] .
\end{gathered}
$$

Though it is hard to determine the sign of the item in the rightmost bracket of (A.7) in general cases, we still can get some conclusions in some special cases. It is clear that if $\lambda_{M} \rightarrow 0$ or $H \rightarrow 0$, then $\partial\left(\pi_{\mathrm{SC}}^{F *}\right) / \partial \tau<0$; if $\lambda_{S} \rightarrow 0$ then $\partial\left(\pi_{\mathrm{SC}}^{F *}\right) / \partial \tau>0$.

Proof of Property 10. (i) According to Tables 1 and 2, when $T<0$,

$$
\begin{aligned}
\frac{\partial \pi_{M}^{F *}}{\partial \lambda_{S}} & =\frac{-\tau\left(1+\tau \lambda_{S}\right)\left[\tau \lambda_{S}+2\left(1+\tau \lambda_{S}\right)(1-\tau) \lambda_{M}+\left(1+\lambda_{M}-\tau \lambda_{M}\right)^{2}\right]}{\left(2+\tau \lambda_{S}\right)^{3}\left[1+\tau \lambda_{S}+(1-\tau) \lambda_{M}\right]^{2}} \frac{\left[a-\beta\left(v_{S}+v_{M}\right)\right]^{2}}{2 \beta}<0, \\
\frac{\partial \pi_{S}^{F *}}{\partial \lambda_{S}} & =\frac{\tau\left(1+\tau \lambda_{S}\right)\left[2(1-\tau) \lambda_{M}-\tau \lambda_{S}\left(1+\tau \lambda_{S}\right)\right]}{\left(2+\tau \lambda_{S}\right)^{3}\left[1+\tau \lambda_{S}+(1-\tau) \lambda_{M}\right]^{2}} \frac{\left[a-\beta\left(v_{S}+v_{M}\right)\right]^{2}}{2 \beta}>0 ;
\end{aligned}
$$

when $T \geq 0$,

$$
\begin{aligned}
& \frac{\partial \pi_{M}^{F *}}{\partial \lambda_{S}}=\frac{-8 \tau\left(1+\lambda_{M}-\tau \lambda_{M}\right)^{2}\left[a-\beta\left(v_{S}+v_{M}\right)\right]^{2}}{\beta\left[4\left(2+\tau \lambda_{S}\right)\left(1+\lambda_{M}-\tau \lambda_{M}\right)-H\right]^{3}\left[1+\tau \lambda_{S}+(1-\tau) \lambda_{M}\right]^{2}} \\
& \quad \times\left[4\left(1+\lambda_{M}-\tau \lambda_{M}\right)\left(1+\tau \lambda_{S}\right)\left[\tau \lambda_{S}+2\left(1+\tau \lambda_{S}\right)(1-\tau) \lambda_{M}\right]+4\left(1+\lambda_{M}-\tau \lambda_{M}\right)^{3}\left(1+\tau \lambda_{S}\right)+\left[(1-\tau) \lambda_{M}\right]^{2} H\right] \\
& \quad<0 \\
& \frac{\partial \pi_{S}^{F *}}{\partial \lambda_{S}}=\frac{-8 \tau\left(1+\lambda_{M}-\tau \lambda_{M}\right)\left[a-\beta\left(v_{S}+v_{M}\right)\right]^{2}}{\beta\left[4\left(2+\tau \lambda_{S}\right)\left(1+\lambda_{M}-\tau \lambda_{M}\right)-H\right]^{3}\left[\left(1+\tau \lambda_{S}\right)+(1-\tau) \lambda_{M}\right]^{2}} \\
& \quad \times\left[4\left(1+\tau \lambda_{S}\right)\left(1+\lambda_{M}-\tau \lambda_{M}\right)^{2}\left[\tau \lambda_{S}\left(1+\tau \lambda_{S}\right)-2(1-\tau) \lambda_{M}\right]\right. \\
& \left.\quad+(1-\tau) \lambda_{M}\left[\left(1+\tau \lambda_{S}\right)^{2}+\left(1+2 \tau \lambda_{S}\right)(1-\tau) \lambda_{M}\right] H\right]
\end{aligned}
$$


Hence, $\partial\left(\pi_{M}^{F *}\right) / \partial \lambda_{S}<0$ always holds no matter whether $T<0$ or $T \geq 0$. While for $\partial\left(\pi_{S}^{F *}\right) / \partial \lambda_{S}$, when $T<0, \partial\left(\pi_{S}^{F *}\right) /$ $\partial \lambda_{S}<0 \Leftrightarrow \tau \lambda_{S}\left(1+\tau \lambda_{S}\right)>2(1-\tau) \lambda_{M}$; when $T \geq 0$, if $\tau \lambda_{S}\left(1+\tau \lambda_{S}\right)>2(1-\tau) \lambda_{M}$, then $\partial\left(\pi_{S}^{F *}\right) / \partial \lambda_{S}<0$; otherwise the sign of $\partial\left(\pi_{S}^{F *}\right) / \partial \lambda_{S}$ is uncertain.

(ii) Also according to Tables 1 and 2, when $T<0$,

$$
\frac{\partial \pi_{S}^{F *}}{\partial \lambda_{M}}=\frac{-(1-\tau)\left(1+\tau \lambda_{S}\right)^{2}}{\left(2+\tau \lambda_{S}\right)^{2}\left[1+\tau \lambda_{S}+(1-\tau) \lambda_{M}\right]^{2}}
$$

$$
\begin{gathered}
\cdot \frac{\left[a-\beta\left(v_{S}+v_{M}\right)\right]^{2}}{2 \beta}<0, \\
\frac{\partial \pi_{M}^{F *}}{\partial \lambda_{M}}=\frac{(1-\tau)\left(1+\tau \lambda_{S}\right)^{2}}{\left(2+\tau \lambda_{S}\right)^{2}\left[1+\tau \lambda_{S}+(1-\tau) \lambda_{M}\right]^{2}} \\
\cdot \frac{\left[a-\beta\left(v_{S}+v_{M}\right)\right]^{2}}{2 \beta}>0 ;
\end{gathered}
$$

when $T \geq 0$,

$$
\begin{aligned}
& \frac{\partial \pi_{S}^{F *}}{\partial \lambda_{M}}=\frac{-8(1-\tau)\left[a-\beta\left(v_{S}+v_{M}\right)\right]^{2}}{\beta\left[4\left(2+\tau \lambda_{S}\right)\left(1+\lambda_{M}-\tau \lambda_{M}\right)-H\right]^{3}\left[\left(1+\tau \lambda_{S}\right)+(1-\tau) \lambda_{M}\right]^{2}} \times\left[4\left(2+\tau \lambda_{S}\right)\left(1+\lambda_{M}-\tau \lambda_{M}\right)^{3}\left(1+\tau \lambda_{S}\right)^{2}\right. \\
& \left.\quad-\left(2+\tau \lambda_{S}\right)\left[\left(1+\tau \lambda_{S}\right)+(1-\tau) \lambda_{M}\right]^{2} H+\left(1+\tau \lambda_{S}\right)^{2}\left(1+\lambda_{M}-\tau \lambda_{M}\right)\left[1+2 \tau \lambda_{S}+(1-\tau) \lambda_{M}\right] H\right] \\
& \quad<-\frac{8(1-\tau)\left(2+\tau \lambda_{S}\right)\left[a-\beta\left(v_{S}+v_{M}\right)\right]^{2}}{\beta\left[4\left(2+\tau \lambda_{S}\right)\left(1+\lambda_{M}-\tau \lambda_{M}\right)-H\right]^{3}}\left[4\left(1+\lambda_{M}-\tau \lambda_{M}\right)-H\right]<0, \\
& \frac{\partial \pi_{M}^{F *}}{\partial \lambda_{M}}=\frac{8(1-\tau)\left(1+\lambda_{M}-\tau \lambda_{M}\right)\left[a-\beta\left(v_{S}+v_{M}\right)\right]^{2}}{\beta\left[4\left(2+\tau \lambda_{S}\right)\left(1+\lambda_{M}-\tau \lambda_{M}\right)-H\right]^{3}\left[\left(1+\tau \lambda_{S}\right)+(1-\tau) \lambda_{M}\right]^{2}} \times\left[4\left(2+\tau \lambda_{S}\right)\left(1+\tau \lambda_{S}\right)^{2}\left(1+\lambda_{M}-\tau \lambda_{M}\right)^{2}\right. \\
& \left.\quad-\left(\left(3+2 \tau \lambda_{S}\right)\left[1+\tau \lambda_{S}+(1-\tau) \lambda_{M}\right]^{2}-\left(1+\tau \lambda_{S}\right)^{2}\left[1+2 \tau \lambda_{S}+(1-\tau) \lambda_{M}\right]\right) H\right] .
\end{aligned}
$$

Hence, $\partial \pi_{S}^{F *} / \partial \lambda_{M}<0$ always holds no matter whether $T<0$ or $T \geq 0$. And when $T<0, \partial \pi_{M}^{F *} / \partial \lambda_{M}>0$ is always true; when $T \geq 0, \partial \pi_{M}^{F *} / \partial \lambda_{M}>0 \Leftrightarrow H<4 \xi \kappa /(\psi+\delta-\kappa)$.

(iii) When $T<0$, from Table 1 ,

$$
\begin{aligned}
& \frac{\partial \pi_{M}^{F *}}{\partial \tau} \\
& \quad=\frac{-\left[a-\beta\left(v_{S}+v_{M}\right)\right]^{2}}{4 \beta\left(2+\tau \lambda_{S}\right)^{3}\left[1+\tau \lambda_{S}+(1-\tau) \lambda_{M}\right]^{2}}\left[\lambda _ { S } \left[\tau \lambda_{S}\right.\right. \\
& \left.\quad+2\left(1+\tau \lambda_{S}\right)(1-\tau) \lambda_{M}\right]\left[1+\tau \lambda_{S}+(1-\tau) \lambda_{M}\right] \\
& \quad+\left(2+\tau \lambda_{S}\right)\left[\lambda_{M}\left(1+\lambda_{S}\right)\left(2+\tau \lambda_{S}\right)+\lambda_{S}\left(1+\lambda_{M}\right)\right. \\
& \left.\left.\quad \cdot\left(1+\tau \lambda_{S}\right)\right]\right]<0, \\
& \frac{\partial \pi_{S}^{F *}}{\partial \tau} \quad \\
& \quad=\frac{\left(1+\tau \lambda_{S}\right)\left[a-\beta\left(v_{S}+v_{M}\right)\right]^{2}}{2 \beta\left(2+\tau \lambda_{S}\right)^{3}\left[1+\tau \lambda_{S}+(1-\tau) \lambda_{M}\right]^{2}}\left\{\lambda_{M}[2(1\right. \\
& \left.\left.\left.\quad+\lambda_{S}\right)+\tau \lambda_{S}\left(1+\tau \lambda_{S}\right)\right]-\lambda_{S}\left(1+\tau \lambda_{S}\right) \tau \lambda_{S}\right\} .
\end{aligned}
$$

Hence, at this time, $\partial \pi_{M}^{F *} / \partial \tau<0$ always holds, while $\partial \pi_{S}^{F *} /$ $\partial \tau>0 \Leftrightarrow \lambda_{M}>\tau \lambda_{S}{ }^{2}\left(1+\tau \lambda_{S}\right) /\left(2\left(1+\lambda_{S}\right)+\tau \lambda_{S}\left(1+\tau \lambda_{S}\right)\right)$.
When $T \geq 0$, for the special cases of $\lambda_{S} \rightarrow 0$ and $\lambda_{M} \rightarrow 0$, we have

$$
\begin{aligned}
& \left.\frac{\partial \pi_{M}^{F *}}{\partial \tau}\right|_{\lambda_{S} \rightarrow 0}=\frac{-8 \lambda_{M}\left[a-\beta\left(v_{M}+v_{S}\right)\right]^{2}}{\beta\left[8\left(1+\lambda_{M}-\tau \lambda_{M}\right)-H\right]^{3}}\{8(1 \\
& \left.\left.+\lambda_{M}-\tau \lambda_{M}\right)-\left[2+3(1-\tau) \lambda_{M}\right] H\right\}, \\
& \left.\frac{\partial \pi_{M}^{F *}}{\partial \tau}\right|_{\lambda_{M} \rightarrow 0}=\frac{-32 \lambda_{S}\left[a-\beta\left(v_{M}+v_{S}\right)\right]^{2}}{\beta\left[4\left(2+\tau \lambda_{S}\right)-H\right]^{3}}<0, \\
& \left.\frac{\partial \pi_{S}^{F *}}{\partial \tau}\right|_{\lambda_{S} \rightarrow 0}=\frac{8 \lambda_{M}\left[a-\beta\left(v_{M}+v_{S}\right)\right]^{2}}{\beta\left[8\left(1+\lambda_{M}-\tau \lambda_{M}\right)-H\right]^{2}}>0, \\
& \left.\frac{\partial \pi_{S}^{F *}}{\partial \tau}\right|_{\lambda_{M} \rightarrow 0}=\frac{-32 \tau \lambda_{S}^{2}\left[a-\beta\left(v_{M}+v_{S}\right)\right]^{2}}{\beta\left[4\left(2+\tau \lambda_{S}\right)-H\right]^{3}}<0 .
\end{aligned}
$$

Thus the results of Property 10(iii) can be derived.

\section{Notations}

$S, M$, SC: Subscripts, denoting the supplier, manufacturer, and supply chain, respectively 


\begin{tabular}{|c|c|}
\hline \multicolumn{2}{|c|}{$C, F, N:$ Superscripts, denoting the settings of } \\
\hline$C, F, N$ : & $\begin{array}{l}\text { integrated supply chain, decentralized one } \\
\text { with fairness-concerned members, and } \\
\text { decentralized one with fairness-neutral } \\
\text { members, }\end{array}$ \\
\hline$a:$ & Potential intrinsic demand \\
\hline$\alpha:$ & Demand sensitivity to quality \\
\hline$\beta:$ & Demand sensitivity to selling price \\
\hline$p:$ & $\begin{array}{l}\text { Selling price per unit of the final products } \\
\text { in the end market }\end{array}$ \\
\hline$q:$ & $\begin{array}{l}\text { Quality of core components provided by } \\
\text { the supplier }\end{array}$ \\
\hline$w:$ & $\begin{array}{l}\text { Wholesale price per unit of the core } \\
\text { components }\end{array}$ \\
\hline$\varepsilon:$ & $\begin{array}{l}\text { The variable production cost parameter } \\
\text { related to quality for the supplier }\end{array}$ \\
\hline$v_{S}:$ & $\begin{array}{l}\text { The variable production cost per unit } \\
\text { irrelevant to quality for the supplier }\end{array}$ \\
\hline$v_{M}:$ & $\begin{array}{l}\text { The variable production cost per unit for } \\
\text { the manufacturer }\end{array}$ \\
\hline$c:$ & $\begin{array}{l}\text { The fixed cost parameter related to quality } \\
\text { for the supplier }\end{array}$ \\
\hline$\lambda_{S}, \lambda_{M}:$ & $\begin{array}{l}\text { The fairness concern coefficient for the } \\
\text { supplier and manufacturer, respectively }\end{array}$ \\
\hline$T:$ & $\begin{array}{l}T:=\alpha-\beta \varepsilon \text {, demand sensitivity to quality } \\
\text { relative to selling price }\end{array}$ \\
\hline$H:$ & $\begin{array}{l}H:=T^{2} / \beta c \text {, the market } \\
\text { innovation-desirability-index } T H\end{array}$ \\
\hline$\tau:$ & The supplier's bargaining power \\
\hline$D$ & The demand for the final products. \\
\hline
\end{tabular}

\section{Conflicts of Interest}

The authors declare that they have no conflicts of interest.

\section{Acknowledgments}

The authors thank the National Natural Science Foundation of China under Grant G71402171, the Natural Science Foundations of Zhejiang province Grant LY14G020004, and the Outstanding Young Fund Key Project of Anhui province under Grant gxyqZD2017088 for supporting this research.

\section{References}

[1] M.-H. Shu and H.-C. Wu, "Quality-based supplier selection and evaluation using fuzzy data," Computers and Industrial Engineering, vol. 57, no. 3, pp. 1072-1079, 2009.

[2] J. Chai, H. Chen, Q. Huang, and W. Yan, "Should a manufacturer encroach on its retailer's operations with quality differentiated products?" Discrete Dynamics in Nature and Society, vol. 2017, Article ID 8367547, 12 pages, 2017.

[3] R. D. Banker, I. Khosla, and K. K. Sinha, "Quality and competition," Management Science, vol. 44, no. 9, pp. 1179-1192, 1998.

[4] G. J. Tellis, E. Yin, and R. Niraj, "How quality drives the rise and fall of high-tech products," MIT Sloan Management Review, vol. 52, no. 4, pp. 14-16, 2011.

[5] J.-C. Li, Y.-W. Zhou, and W. Huang, "Production and procurement strategies for seasonal product supply chain under yield uncertainty with commitment-option contracts," International Journal of Production Economics, vol. 183, pp. 208-222, 2017.

[6] W. Xu, Y. Yu, and Q. Zhang, "An evaluation method of comprehensive product quality for customer satisfaction based on intuitionistic fuzzy number," Discrete Dynamics in Nature and Society, vol. 2018, Article ID 5385627, 12 pages, 2018.

[7] H. Gurnani, M. Erkoc, and Y. Luo, "Impact of product pricing and timing of investment decisions on supply chain coopetition," European Journal of Operational Research, vol. 180, no. 1, pp. 228-248, 2007.

[8] C. D. Looper, "Apple vs. Qualcomm: Everything you need to know," 2017, https://www.digitaltrends.com/business/applevs-qualcomm-news/.

[9] D. Kahneman, J. L. Knetsch, and R. H. Thaler, "Fairness and the assumptions of economics," The Journal of Business, vol. 59, no. S4, pp. S285-S300, 1986.

[10] E. Fehr and K. M. Schmidt, "A theory of fairness, competition, and cooperation," The Quarterly Journal of Economics, vol. 114, no. 3, pp. 817-868, 1999.

[11] N. Kumar, "The power of trust in manufacturer-retailer relationships," Harvard Business Review, vol. 74, no. 6, pp. 92-106, 1996.

[12] L. K. Scheer, N. Kumar, and J.-B. E. M. Steenkamp, "Reactions to perceived inequity in U.S. and Dutch interorganizational relationships," Academy of Management Journal (AMJ), vol. 46, no. 3, pp. 303-316, 2003.

[13] C. H. Loch and Y. Z. Wu, "Social preferences and supply chain performance: an experimental study," Management Science, vol. 54, no. 11, pp. 1835-1849, 2008.

[14] E. Katok and V. Pavlov, "Fairness in supply chain contracts: a laboratory study," Journal of Operations Management, vol. 31, no. 3, pp. 129-137, 2013.

[15] N. Matsubayashi, "Price and quality competition: the effect of differentiation and vertical integration," European Journal of Operational Research, vol. 180, no. 2, pp. 907-921, 2007.

[16] N. Matsubayashi and Y. Yamada, "A note on price and quality competition between asymmetric firms," European Journal of Operational Research, vol. 187, no. 2, pp. 571-581, 2008.

[17] J. Yu and S. Ma, "Impact of decision sequence of pricing and quality investment in decentralized assembly system," Journal of Manufacturing Systems, vol. 32, no. 4, pp. 664-679, 2013.

[18] M. Seifbarghy, K. Nouhi, and A. Mahmoudi, "Contract design in a supply chain considering price and quality dependent demand with customer segmentation," International Journal of Production Economics, vol. 167, pp. 108-118, 2015.

[19] S. Wang, Q. Hu, and W. Liu, "Price and quality-based competition and channel structure with consumer loyalty," European Journal of Operational Research, vol. 262, no. 2, pp. 563-574, 2017.

[20] G. Xie, S. Wang, and K. K. Lai, "Quality improvement in competing supply chains," International Journal of Production Economics, vol. 134, no. 1, pp. 262-270, 2011.

[21] G. Xie, W. Yue, S. Wang, and K. K. Lai, "Quality investment and price decision in a risk-averse supply chain," European Journal of Operational Research, vol. 214, no. 2, pp. 403-410, 2011.

[22] B. C. Giri, B. Roy, and T. Maiti, "Coordinating a three-echelon supply chain under price and quality dependent demand with sub-supply chain and RFM strategies," Applied Mathematical Modelling: Simulation and Computation for Engineering and Environmental Systems, vol. 52, pp. 747-769, 2017. 
[23] J. Chen, L. Liang, D.-Q. Yao, and S. Sun, "Price and quality decisions in dual-channel supply chains," European Journal of Operational Research, vol. 259, no. 3, pp. 935-948, 2017.

[24] T. H. Cui, J. S. Raju, and Z. J. Zhang, "Fairness and channel coordination," Management Science, vol. 53, no. 8, pp. 1303-1314, 2007.

[25] Y. Chen and T. H. Cui, "The benefit of uniform price for branded variants," Marketing Science, vol. 32, no. 1, pp. 36-50, 2013.

[26] K. J. Li and S. Jain, "Behavior-based pricing: An analysis of the impact of peer-induced fairness," Management Science, vol. 62, no. 9, pp. 2705-2721, 2016.

[27] S. Du, L. Wei, Y. Zhu, and T. Nie, "Peer-regarding fairness in supply chain," International Journal of Production Research, pp. 1-13, 2016.

[28] T. Nie and S. Du, "Dual-fairness supply chain with quantity discount contracts," European Journal of Operational Research, vol. 258, no. 2, pp. 491-500, 2017.

[29] E. Katok, T. Olsen, and V. Pavlov, "Wholesale pricing under mild and privately known concerns for fairness," Production Engineering Research and Development, vol. 23, no. 2, pp. 285302, 2014.

[30] Q.-H. Li and B. Li, "Dual-channel supply chain equilibrium problems regarding retail services and fairness concerns," Applied Mathematical Modelling, vol. 40, no. 15-16, pp. 73497367, 2016.

[31] T.-H. Ho, X. Su, and Y. Wu, "Distributional and peer-induced fairness in supply chain contract design," Production Engineering Research and Development, vol. 23, no. 2, pp. 161-175, 2014.

[32] S. F. Du, T. F. Nie, C. B. Chu, and Y. Yu, "Newsvendor model for a dyadic supply chain with Nash bargaining fairness concerns," International Journal of Production Research, vol. 52, no. 17, pp. 5070-5085, 2014.

[33] J. Chen, Y.-W. Zhou, and Y. Zhong, "A pricing/ordering model for a dyadic supply chain with buyback guarantee financing and fairness concerns," International Journal of Production Research, vol. 55, no. 18, pp. 5287-5304, 2017.

[34] X. Guo and B. Jiang, "Signaling through price and quality to consumers with fairness concerns," Journal of Marketing Research, vol. 53, no. 6, pp. 988-1000, 2016.

[35] K. Jerath, S.-H. Kim, and R. Swinney, "Product quality in a distribution channel with inventory risk," Marketing Science, vol. 36, no. 5, pp. 747-761, 2017.

[36] P. Nelson, "Information and consumer behavior," Journal of Political Economy, vol. 78, no. 2, pp. 311-329, 1970.

[37] J. Nash, “The bargaining problem," Econometrica: Journal of the Econometric Society, vol. 18, no. 2, pp. 155-162, 1950.

[38] J. C. Harsanyi and R. Selten, "A generalized Nash solution for two-person bargaining games with incomplete information," Management Science, vol. 18, pp. P80-P106, 1972.

[39] G. Charness and M. Rabin, "Understanding social preferences with simple tests," The Quarterly Journal of Economics, vol. 117, no. 3, pp. 817-869, 2002.

[40] Y.-W. Zhou, J. Li, and Y. Zhong, "Cooperative advertising and ordering policies in a two-echelon supply chain with risk-averse agents," Omega, vol. 75, pp. 97-117, 2018. 


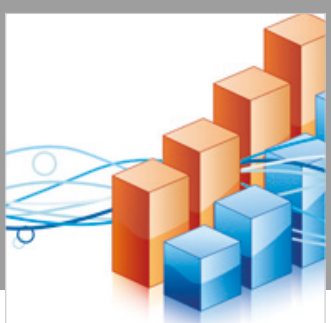

Advances in

Operations Research

\section{-n-m}
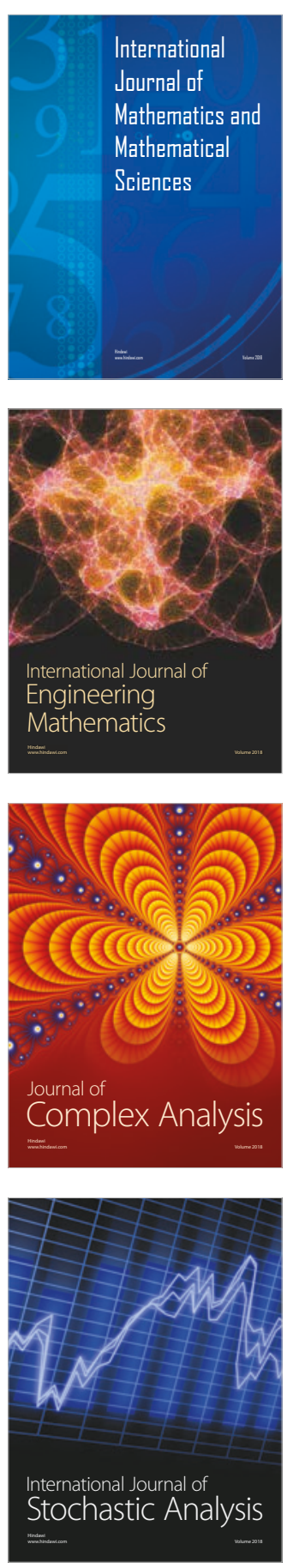
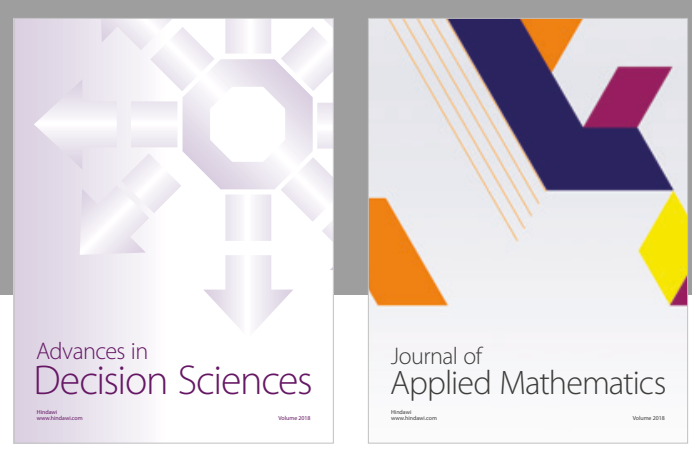

Journal of

Applied Mathematics
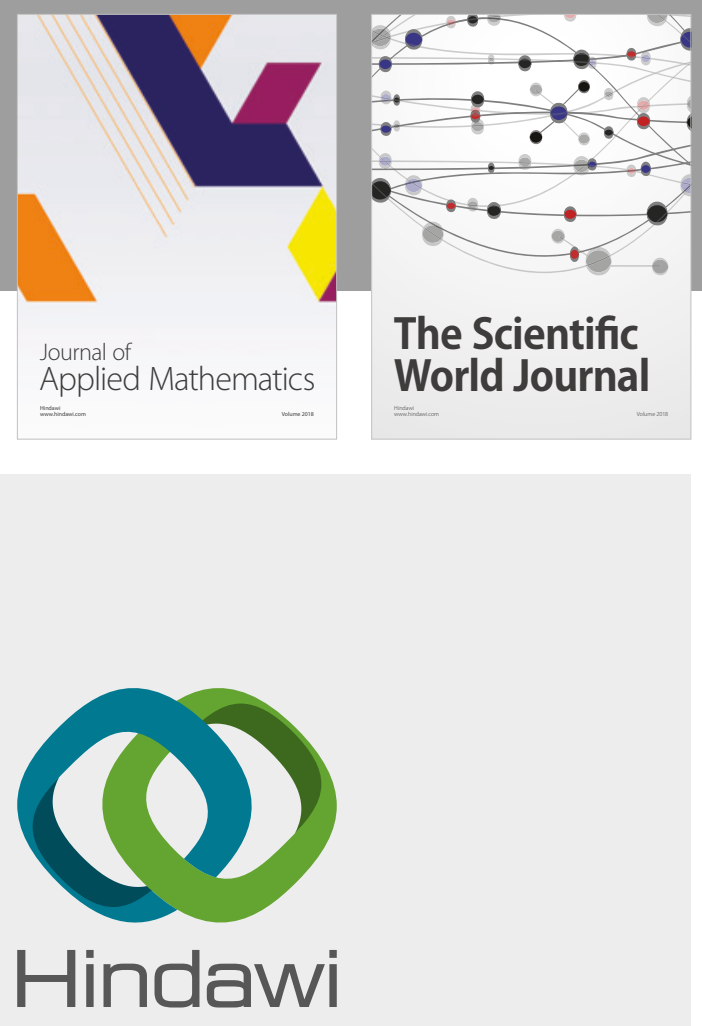

Submit your manuscripts at

www.hindawi.com

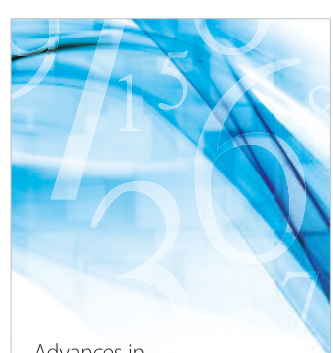

Advances in
Numerical Analysis
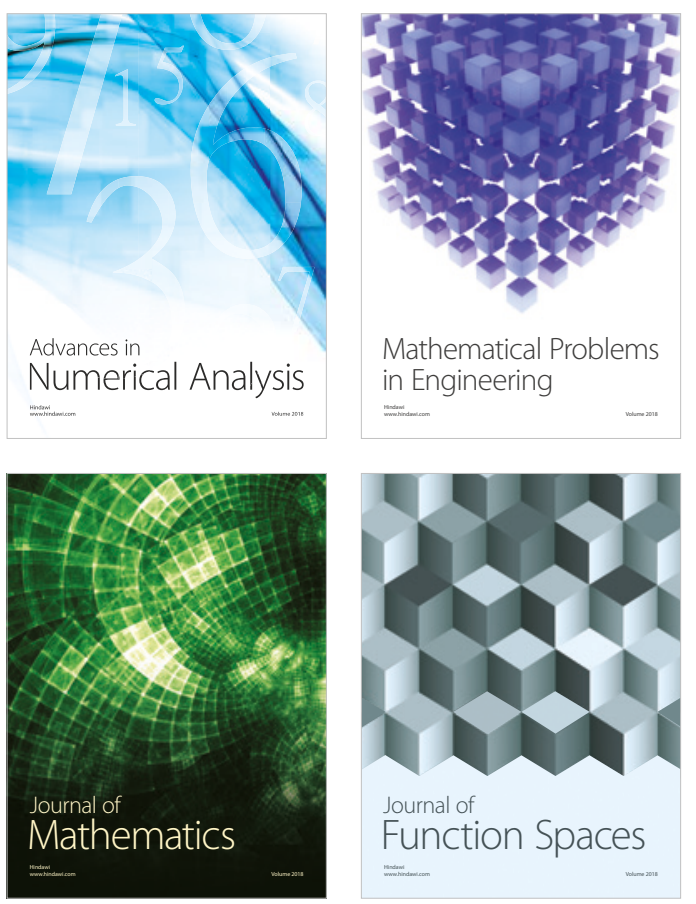

Mathematical Problems in Engineering

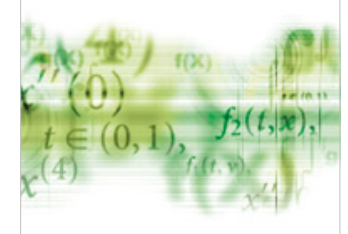

International Journal of

Differential Equations

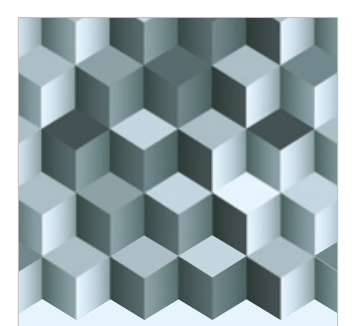

Journal of

Function Spaces

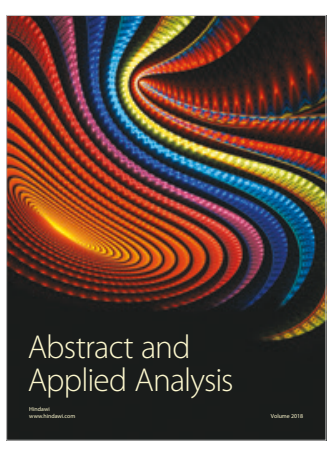

The Scientific

World Journal

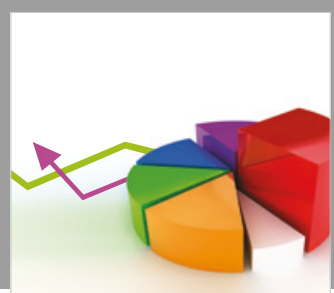

Journal of

Probability and Statistics
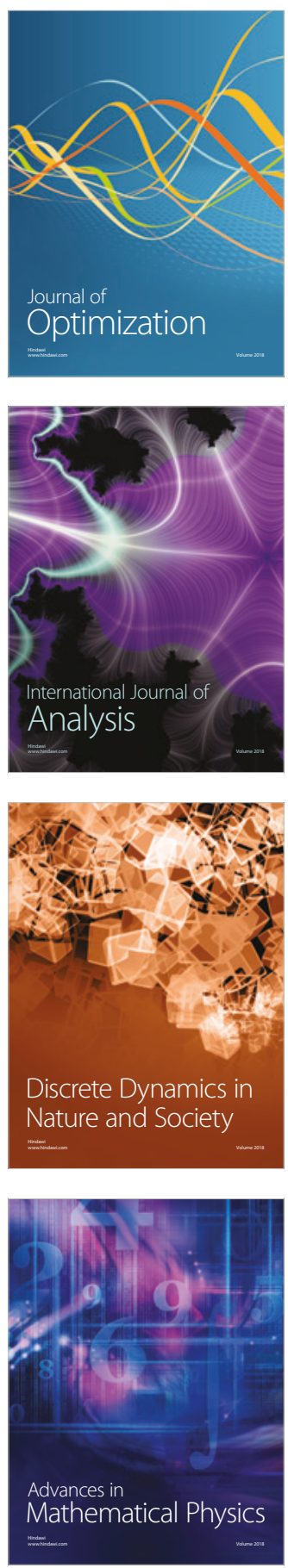